\title{
Deformations of Flexible and Foldable Electro-active Composite Structures
}

\author{
Vahid Tajeddini and Anastasia Muliana* \\ Department of Mechanical Engineering, Texas A\&M University, College Station, United States \\ * Corresponding author: amuliana@tamu.edu
}

\begin{abstract}
This paper presents numerical analyses of elastic and viscoelastic smart flexible and foldable composite structures under electric field actuation. The studied composites comprise of multiple distributed piezoelectric patches bonded to the surfaces of in-active thin planar structures (substrates). Upon applications of electric field input, the planar structures can undergo three-dimensional large rotational deformations while their strains and stretches remain relatively small. A nonlinear time-dependent electro-mechanical coupling relation for the piezoelectric patches is considered to simulate more precisely response of piezoelectric materials when subjected to large magnitude of electric field. Co-rotational Lagrangian finite element approach is used for solving the governing equations of the deformations of flexible and foldable electroactive composite structures. Various three-dimensional shape changes of originally planar structures are achieved with different arrangements of integrated patches and subjected to different magnitude of electric fields. The effect of viscoelastic substrates and time-dependent electro-mechanical coupling of piezoelectric materials on the deformed shapes is also studied. This analysis can help designers in simulating desired deformed shapes and determining external stimuli to be prescribed prior to fabricating smart and flexible composites.
\end{abstract}




\section{Introduction}

Structures integrated with smart materials, such as shape memory, magnetostrictive and piezoelectric materials, known as smart structures, are appealing for the development of new generation of autonomous systems with sensing and adaptation ability. Smart structures that are lightweight, flexible and deformable into various configurations find many applications in aerospace industry, biomedicine and robotics. Examples of applications are morphing wings, micro air vehicles, soft robots, artificial skins, vascular stents, and electronic packaging systems. Flexible structures are macroscopically compliant, which are often in the forms of long filaments and thin sheets that can be deformed into various geometrical shapes. Depending on the geometrical shapes considered, flexible structures can experience relatively small strains while undergoing large rotations $[1,2]$. In this work, we study the deformation of smart flexible planar structures with integrated thin piezoelectric patches, in which deformations and shape changes are controlled by electric field inputs prescribed to the piezoelectric actuators. We consider two piezoelectric materials for the patches, which are, lead zirconate titanate (PZT) ceramics and active fiber composites (AFC). When piezoelectric ceramics based actuators are considered, it limits the strains in the flexible structures and large deformations in such structures are predominantly due to large rotations. One solution to enhance deformations in piezoelectric ceramics based actuators is to form active fiber composites (AFC) which have PZT fibers embedded in a polymeric matrix. AFC was originally proposed by Ben and Hagood [3].

Flexible structures are typically undergoing large displacements (large stretch and/or large rotation) when subjected to external stimuli, whose motion cannot be sufficiently described based on linearized kinematics. There have been several studies on analyzing three-dimensional deformation of thin plates and shells with nonlinear geometries [4-6] mainly under mechanical loads. Moderate and large deformations of plates are governed by coupled nonlinear differential equations for which analytical solutions are available only for a few cases involving simple geometries and loading conditions [7]. There have been limited studies on analyzing nonlinear deformations of plates and shells integrated with piezoelectric actuator. Chen and Chen [8] studied piezoelectric layered-plates by adopting von Karman theory and using finite difference method in order to obtain solutions to the governing equations for moderate deformations, e.g. von Karman strains. Xue et al. [9] solved nonlinear partial differential equations for moderate 
deflections of thin plates made of piezoemagnetic and piezoelectric materials under transverse mechanical loads by using Bubnov-Galerkin method.

One of the commonly used numerical techniques for solving large deformations of plates and shells is finite element method (FEM). In FE analysis with geometric nonlinearity, three Lagrangian kinematic descriptions have been formulated: (a) total Lagrangian (TL), (b) updated Lagrangian (UL) and (c) co-rotational (CR). For the TL approach, the governing equations are written with respect to the original configuration of the body while for the UL method, the reference configuration is updated at each step or increment, and the equations are formulated with respect to the equilibrated configuration from the previous step. The CR description is based on a separation of rigid body displacements from the deformational displacements, which can be used for analyzing structures undergoing large deformation mainly due to rotations while the strains remain relatively small. In the $\mathrm{CR}$ method, the deformational component is typically formed based on small strain measures, although other general strain measures are also possible. The governing equations are expressed with respect to the current configuration obtained from the rigid body motion of the original configuration. The CR formulation was first introduced by Wempner [10] in 1969 and Belytschko et al. [11] in 1973. Since then, it has been adopted by researchers for studying deformation of structures and multi-body systems which often involves large ratio of rigid body motions to total deformation [12-16]. Felippa and Haugen [17] summarized the existing CR formulations and presented a unified theoretical framework for corotational finite element (CRFE) in geometrically nonlinear analyses of structures. Recently, by using the CR formulation, triangular flat shell elements were constructed by researchers for nonlinear analysis of shells and plates subjected to small strains and large rotations due to mechanical loads [18-20]. Cai and Atluri [21,22] considered also moderate strains by adopting the von-Karman nonlinear strains in the rotated element frame. In all of the above studies, the CR formulation has been considered for flexible linear elastic bodies under mechanical loadings. To the best of our knowledge, the CRFE method has not been used for studying deformations in flexible elastic as well as viscoelastic composite plates/shells actuated by non-mechanical stimuli.

In actuation applications, it is often necessary to apply high electric field inputs to the piezoelectric components in order to obtain large deformations. When subjected to high electric fields, the piezoelectric materials often experience nonlinear electro-mechanical responses. 
Tiersten [23] was among researchers who studied nonlinear electro-mechanical behaviors of polarized piezoelectric ceramics. He formulated an electro-mechanical constitutive model by considering higher order terms of the electric field in order to describe the nonlinear electromechanical coupling behavior of piezoelectric ceramics. A limited number of studies have considered nonlinear electro-mechanical response of piezoelectric materials due to large electric fields [24-26], but for small deformations. Mollayousef [27] and Ben Atitallah et al. [28, 29] have studied material characterization of AFC under a wider range of electro-mechanical inputs. Mollayousef used a unit cell approach to characterize the electro-mechanical properties of AFC. Ben Atitallah et al. measured the material properties of AFCs and showed nonlinear time and temperature dependent response of the composite under high temperatures and different strain rates as well as high electric voltages. Tajeddini et al. [30] modeled the relaxation behaviors of AFCs and predicted the creep response of the material under different temperatures and stress levels with a quasi linear viscoelastic (QLV) approach.

In many flexible structures, polymers are widely used for the substrate because of their capability in undergoing large deformations and they are generally lightweight. One of the prominent characteristics of polymers is their viscoelastic behavior. It might be necessary to consider the time-dependent behaviors in the viscoelastic polymeric structures when nonmechanical stimuli are prescribed in order to obtain shape changes. In this study, 3D deformations of smart composite structures having polymeric substrates integrated with piezoelectric patches undergoing large deformations are studied. Co-rotational finite element (CRFE) method is used for numerically solving the equations that govern the deformations of the electro-active composites. Both linear elastic and linear viscoelastic behaviors are considered for the substrates and patches. Nonlinear time-dependent electro-mechanical constitutive equation is considered for the active piezoelectric materials. The numerical results of the deformations and shape changes of smart composite structures are presented. The manuscript is organized as follows. Section 2 presents general governing equations of the nonlinear deformations in electroactive composites. Both piezoelectric ceramics and AFCs are considered as actuators. Section 3 discusses CRFE method for nonlinear structural analysis. Section 4 discussed the CRFE method for the electro-active composite plates followed by numerical analyses of composites plates under various boundary conditions. The study is wrapped up in Section 5. 


\section{Governing Equations of the Nonlinear Deformations in Electro-active Composite Plates}

\subsection{Piezoelectric ceramic actuators on viscoelastic substrates}

The undeformed structure is considered in the shape of slender flat planar structures which can undergo 3D shape changes. Multiple patches with arbitrary arrangement can be bonded to the top and bottom surfaces of the substrate symmetrically with respect to the middle plane of the plate. It is assumed that the patches are perfectly bonded to the plates. The structure is assumed to have a uniform thickness. Dimitriadis et al. [31] calculated the bending load induced to an elastic plate by a pair of electrically stimulated homogeneous piezoelectric patches bonded perfectly on its top and bottom. For the elastic plates, we follow a similar approach and present the formulation for more general case where the piezoelectric patches are thin and orthotropic. Then, we take into account time-dependent material properties for the substrate and adopt the Laplace transformed method for solving governing equations in case of viscoelastic substrates are considered.

Consider a differential plate element with thickness $2 h$ having integrated actuators at the top and bottom faces each with thickness $t$ as shown in Figure 1. Since the patches are assumed perfectly bonded to the homogeneous substrate, the displacements at the interfaces of the substrate and the piezoelectric patches are continuous; and due to the differences in the elastic moduli of the patches and substrate, stress discontinuities arise at the interfaces. Figure 1 represents the $x-z$ and $y-z$ stress distributions. The electric stimulus is prescribed on the top and bottom surfaces of the plate such that it induces bending moments to the plate. Therefore, the stress distributions due to the electric stimulus prescribed on the top and bottom actuators must be anti-symmetric about the neutral axis in order to create bending moments and curvatures. The normal stress distributions within the plate resulting from the couples $m_{x}$ and $m_{y}$ per unit length (bending moments) are:

$$
\begin{aligned}
& \int_{-h}^{h} \sigma_{x} z d y d z=m_{x} d y \\
& \int_{-h}^{h} \sigma_{y} z d x d z=m_{y} d x
\end{aligned}
$$


As we are dealing with thin plates, the transverse shear deformations are considered negligible. The axial in-plane strain distribution is considered small and varies linearly along the thickness direction, which is expressed as:

$$
\varepsilon_{x}=z \kappa_{x}, \quad \varepsilon_{y}=z \kappa_{y}
$$

where $\kappa_{x}$ and $\kappa_{y}$ are the curvatures of the neutral surface of the plate parallel to the $x-z$ and $y-z$ planes. Considering an isotropic and linear elastic constitutive model for the substrate, the stressstrain relations at the interface for the linear elastic plate is

$$
\begin{aligned}
& \left(\sigma_{x i}\right)_{s}=\frac{E_{s}}{1-v_{s}^{2}}\left(\varepsilon_{x i}+v_{s} \varepsilon_{y i}\right) \\
& \left(\sigma_{y i}\right)_{s}=\frac{E_{s}}{1-v_{s}^{2}}\left(\varepsilon_{y i}+v_{s} \varepsilon_{x i}\right)
\end{aligned}
$$

where the axial strains are considered continuous at the interface of the patches and substrates. The subscript $i$ of the stresses and strains denotes their quantities at the interface and the subscripts $p$ and $s$ denote the quantities of the piezoelectric material and the substrate, respectively. $E$ is the elastic modulus and $v$ is the Poisson ratio. The stress-strain relations of the actuator at the interface is expressed as

$$
\begin{aligned}
& \left(\sigma_{x i}\right)_{p}=\frac{E_{p}}{1-v_{p}^{2}}\left(\varepsilon_{x i}+v_{p} \varepsilon_{y i}-\left(\varepsilon_{x}\right)_{p}-v_{p}\left(\varepsilon_{y}\right)_{p}\right) \\
& \left(\sigma_{y i}\right)_{p}=\frac{E_{p}}{1-v_{p}^{2}}\left(\varepsilon_{y i}+v_{p} \varepsilon_{x i}-\left(\varepsilon_{y}\right)_{p}-v_{p}\left(\varepsilon_{x}\right)_{p}\right)
\end{aligned}
$$

where $\left(\varepsilon_{x}\right)_{p}$ and $\left(\varepsilon_{y}\right)_{p}$ denote the unconstrained free strains in the $x$-and $y$-directions, respectively. These strains are due to the application of electric fields to the piezoelectric patches, which are defined in terms of the applied electric field as follows

$$
\begin{gathered}
\varepsilon_{p}=d_{13} E^{e} \\
\varepsilon_{p}=d_{13} E^{e}+\frac{1}{2} \beta_{13} E^{e 2}
\end{gathered}
$$


where $d_{13}$ is linear piezoelectric constant and $E^{e}$ is electric field in the through thickness direction, $z$, of the patch, $\beta_{13}$ is the higher order piezoelectric constant. The linear electromechanical constitutive relation, Eq. (5-a) is valid for a relatively small electric field input and the second order term of the electric field is included for relatively large electric field input, Eq. $(5-b)$.

By assuming a linear strain variation in the thickness direction of the plate and the patches, and imposing the moment equilibrium about the neutral axis of the plate in $x$ - and $y$ directions, the interface strains can be derived from solving the following system of linear equations:

$$
\begin{aligned}
& A\left(\varepsilon_{x i}+v_{s} \varepsilon_{y i}\right)=B\left(E_{p} \varepsilon_{x i}+v_{p} E_{p} \varepsilon_{y i}-E_{p}\left(1+v_{p}\right) \varepsilon_{p}\right) \\
& A\left(\varepsilon_{y i}+v_{s} \varepsilon_{x i}\right)=B\left(E_{p} \varepsilon_{y i}+v_{p} E_{p} \varepsilon_{x i}-E_{p}\left(1+v_{p}\right) \varepsilon_{p}\right)
\end{aligned}
$$

where for the elastic substrate

$$
A=\frac{E_{s}}{1-v_{s}^{2}} ; B=\frac{-3 h t(2 h+t)}{\left(2 h^{3}+3 h t^{2}+2 t^{3}\right)\left(1-v_{s}^{2}\right)}
$$

From Eq. (6), the strains $\varepsilon_{x i}$ and $\varepsilon_{y i}$ are determined, and the couples $m_{x}$ and $m_{y}$ are then obtained as follows

$$
\begin{aligned}
& m_{x}=\frac{2 h^{2}}{3}\left(\frac{E_{s}}{1-v_{s}^{2}}\right)\left(\varepsilon_{x i}+v_{s} \varepsilon_{y i}\right) \\
& m_{y}=\frac{2 h^{2}}{3}\left(\frac{E_{s}}{1-v_{s}^{2}}\right)\left(\varepsilon_{y i}+v_{s} \varepsilon_{x i}\right)
\end{aligned}
$$

When the substrate is viscoelastic but the patches are elastic, by considering a linear viscoelastic constitutive relation for the substrate subjected to small strains under a timedependent electric field stimulus, Eqs. (6) are rewritten as 


$$
\begin{aligned}
& \int_{0}^{t} A(t-\tau) \frac{d \varepsilon_{x i}}{d \tau} d \tau+v_{p l} \int_{0}^{t} A(t-\tau) \frac{d \varepsilon_{y i}}{d \tau} d \tau=B\left(E_{p} \varepsilon_{x i}(t)+v_{p} E_{p} \varepsilon_{y i}(t)-E_{p}\left(1+v_{p}\right) \varepsilon_{p}(t)\right) \\
& \int_{0}^{t} A(t-\tau) \frac{d \varepsilon_{y i}}{d \tau} d \tau+v_{p l} \int_{0}^{t} A(t-\tau) \frac{d \varepsilon_{x i}}{d \tau} d \tau=B\left(E_{p} \varepsilon_{y i}(t)+v_{p} E_{p} \varepsilon_{x i}(t)-E_{p}\left(1+v_{p}\right) \varepsilon_{p}(t)\right)
\end{aligned}
$$

$A$ is time-dependent because of its dependency on the relaxation function of the substrate, $E_{s}(t)$. It is assumed that the Poisson effect is constant. The free strain of the elastic piezoelectric material $\varepsilon_{p}$ is defined as before which is a function of time if the electric stimulus changes over time. In a more concise form, Eq. (9) is written as

$$
\begin{aligned}
& A(t) * \mathrm{D} \varepsilon_{x i}(t)+v_{s} A(t) * \mathrm{D} \varepsilon_{y i}(t)=B\left(E_{p} \varepsilon_{x i}(t)+v_{p} E_{p} \varepsilon_{y i}(t)-E_{p}\left(1+v_{p}\right) \varepsilon_{p}(t)\right) \\
& A(t) * \mathrm{D} \varepsilon_{y i}(t)+v_{s} A(t) * \mathrm{D} \varepsilon_{x i}(t)=B\left(E_{p} \varepsilon_{y i}(t)+v_{p} E_{p} \varepsilon_{x i}(t)-E_{p}\left(1+v_{p}\right) \varepsilon_{p}(t)\right)
\end{aligned}
$$

where D denotes the time derivative operator and ' $*$ 'stands for the convolution integral over [0, $t$ ]. In order to solve a set of integral-differential equations, the Laplace transformed method is used to transfer the system of integral equations (6) to Laplace domain and obtain the following algebraic equations

$$
\begin{aligned}
& \tilde{A} \tilde{\mathrm{D}} \varepsilon_{x i}+v_{s} \tilde{A} \tilde{\mathrm{D}} \varepsilon_{y i}=B\left(E_{p} \tilde{\mathrm{D}} \varepsilon_{x i}+v_{p} E_{p} \tilde{\mathrm{D}} \varepsilon_{y i}-E_{p}\left(1+v_{p}\right) \tilde{\mathrm{D}} \varepsilon_{p}\right) / s \\
& \tilde{A} \tilde{\mathrm{D}} \varepsilon_{y i}+v_{s} \tilde{A} \tilde{\mathrm{D}} \varepsilon_{x i}=B\left(E_{p} \tilde{\mathrm{D}} \varepsilon_{x i}+v_{p} E_{p} \tilde{\mathrm{D}} \varepsilon_{y i}-E_{p}\left(1+v_{p}\right) \tilde{\mathrm{D}} \varepsilon_{p}\right) / s
\end{aligned}
$$

where superscript ' $\sim$ ' indicates that the time-dependent function in Laplace domain. By solving the above equations and then by taking inverse of the Laplace transform, the time-dependent interface strains are determined and then in a similar way to the elastic case, time-dependent induced moments to the substructure are derived.

\subsection{AFC actuators on the polymeric substrates}

As discussed above in order to create more flexible piezoelectric ceramics actuators, AFCs have been considered. The AFCs used in this study are from Advanced Cerametrics Inc. and the material parameters are obtained from Ben Attitalah et al. [28] and Tajeddini et al. [30]. In these experimental studies, AFCs are shown to experience time-dependent electro-mechanical responses. 
The time-dependent relaxation function of the AFC in the fiber direction, $x$, is assumed to have the following form:

$$
E_{p, x x}(t)=E_{p, x x} e l\left(\Upsilon_{\infty}+\sum_{n=1}^{N} \Upsilon_{n} e^{-t / \tau_{n}}\right)
$$

where $\Upsilon_{\infty}+\sum_{n=1}^{N} \Upsilon_{n}=1$ and $\Upsilon_{\infty}+\sum_{n=1}^{N} \Upsilon_{n} e^{-t / \tau_{n}}$ is called the normalized relaxation function. Under a uniaxial tensile test in the fiber direction, the tensile elastic modulus of the AFC in the fiber direction $E_{p x x}^{e l}$ is measured as $9.76 \mathrm{GPa}$. Figure 2 shows the relaxation data from experiment and the fitted curve based on the Prony series of Eq. (12). The calibrated parameters are given in Table 1. For the piezoelectric coefficients of the AFC, the values reported by Khan et al. [32] are used. The linear piezoelectric coefficient in the fiber direction $\left(d_{11}\right)$ of the AFC is expressed as a time-dependent kernel function given in Eq. (13) and the material parameters are shown in Table 2.

$$
d_{p x x}(t)=d_{p x x, 0}+\sum_{n=1}^{N} d_{p x x, n}\left[1-e^{-t / \lambda_{n}}\right]
$$

Based on available experimental data, the nonlinear piezoelectric coefficient $\left(\beta_{\mathrm{p} 11}\right)$ is assumed to have similar time-dependent function and differs from the linear coefficient by a constant, which is calibrated from an instantaneous response of the piezoelectric material. This approach is similar to the quasi-linear viscoelastic model, originally proposed by Fung [33]. Figure 3 shows the experimental data and estimated data from least square algorithm. The calibrated properties are $\quad \varepsilon=d_{p x x, 0}\left(E^{e}+2.32 \times 10^{-5} E^{e 2}\right) \quad$ and therefore, $\beta_{p x x}=2.32 \times 10^{-5}(m / V) \times d_{p 11}$.

The AFC is considered orthotropic with fibers in $x$-direction. Due to lack of experimental data, the properties in directions perpendicular to the fibers are approximated as $E_{p y y}(t) \approx(15 / 19) E_{p x x}(t) \quad[32]$ and $d_{y x}(t) \approx-0.5 d_{x x}(t)[34]$. The corresponding Poisson's coefficients $\left(v_{x y}=0.28\right.$ and $\left.v_{y x}=(15 / 19) v_{x y}\right)$, from [32], of the material are assumed timeindependent. 
The stress-strain relations for the AFC incorporating time-dependent electro-mechanical effect are written as:

$$
\begin{aligned}
& \left(\sigma_{x i}\right)_{p}=\frac{1}{1-v_{x y} v_{y x}}\left(E_{p x} * \mathrm{D} \varepsilon_{x i}+v_{12} E_{p y} * \mathrm{D} \varepsilon_{y i}-E_{p x} * \mathrm{D} \varepsilon_{p x}-v_{12} E_{p y} * \mathrm{D} \varepsilon_{p y}\right), \\
& \left(\sigma_{y i}\right)_{p}=\frac{1}{1-v_{x y} v_{y x}}\left(E_{p y} * \mathrm{D} \varepsilon_{y i}+v_{12} E_{p y} * \mathrm{D} \varepsilon_{x i}-E_{p x} * \mathrm{D} \varepsilon_{p y}-v_{12} E_{p y} * \mathrm{D} \varepsilon_{p x}\right)
\end{aligned}
$$

From the equilibrium conditions and considering the continuity condition at the interface, the following sets of equations in terms of unknowns strains $\varepsilon_{y i}$ and $\varepsilon_{x i}$ are:

$$
\begin{aligned}
& \left(\frac{E_{s}(t)}{1-v_{p l}^{2}}-\frac{\kappa_{g} E_{p x}(t)}{1-v_{x y} v_{y x}}\right) * \mathrm{D} \varepsilon_{x i}(t)+\left(\frac{v_{s} E_{s}(t)}{1-v_{s}^{2}}-\frac{\kappa_{g} v_{x y} E_{p y}(t)}{1-v_{x y} v_{y x}}\right) * \mathrm{D} \varepsilon_{y i}(t)= \\
& \frac{\kappa_{g}}{1-v_{x y} v_{y x}}\left(-E_{p x}(t) * \mathrm{D} \varepsilon_{p x}(t)-v_{12} E_{p y}(t) * \mathrm{D} \varepsilon_{p y}(t)\right), \\
& \left(\frac{E_{s}(t)}{1-v_{p l}^{2}}-\frac{\kappa_{g} E_{p y}(t)}{1-v_{x y} v_{y x}}\right) * \mathrm{D} \varepsilon_{y i}(t)+\left(\frac{v_{s} E_{s}(t)}{1-v_{s}^{2}}-\frac{\kappa_{g} v_{x y} E_{p y}(t)}{1-v_{x y} v_{y x}}\right) * \mathrm{D} \varepsilon_{x i}(t)= \\
& \frac{\kappa_{g}}{1-v_{x y} v_{y x}}\left(-E_{p y}(t)^{*} \mathrm{D} \varepsilon_{p y}(t)-v_{x y} E_{p y}(t)^{*} \mathrm{D} \varepsilon_{p x}(t)\right)
\end{aligned}
$$

where

$$
\begin{aligned}
& \varepsilon_{p x}=\int_{0}^{t} d_{x x}(t-\tau) \frac{\partial F^{\varepsilon}}{\partial E_{x}^{e}} \frac{\partial E_{1}^{e}(\tau)}{\partial \tau} d \tau \\
& \varepsilon_{p y}=\int_{0}^{t} d_{y x}(t-\tau) \frac{\partial F^{\varepsilon}}{\partial E_{y}^{e}} \frac{\partial E_{1}^{e}(\tau)}{\partial \tau} d \tau \quad(x \text { is in fiber direction) }
\end{aligned}
$$

and the nonlinear electric field measure in Eq. (16-a) is given as

$$
F^{\varepsilon}=E_{x}^{e}+2.32 \times 10^{-5}(m / V) E_{x}^{e 2}
$$

By solving Eq. (15) in Laplace domain, the induced time-dependent moments to the substrate can be determined. 


\section{CRFE Methods for Nonlinear Deformations of Electro-active Composite Structures}

Using the CRFE approach, 3D deformations of smart thin planar structures undergoing small strains but large rotations due to electro-mechanical input are studied. The CR formulation is based upon an explicit separation of rigid body motions, including translations and rotations, from the total deformations. The benefit gained through this approach is that an existing linear finite element method is used for the deformational part of motion, while a nonlinear analysis is incorporated for the rigid body motions. The CRFE formulation is documented in details in [20, 35]. A summary of the approach and main formulas required in the present analyses are discussed as follows.

The kinematics of CR formulation is shown for an element in Figure 4. The element in its initial configuration is denoted by $C^{0}$. The motion of the element under an applied load transforms the body to a deformed configuration $C^{\mathrm{D}}$. The rigid-body motion experienced by the element brings the initial configuration to the co-rotated configuration $C^{\mathrm{R}}$ (shown in dashed line).

Two types of coordinate systems are considered: global coordinate system defined by the triad of unit orthogonal vectors $(i, j, k)$ and local coordinate system of the element including the initial coordinate system $\left(\mathbf{e}_{1}^{0}, \mathbf{e}_{2}^{0}, \mathbf{e}_{3}^{0}\right)$ and co-rotated coordinate system $\left(\mathbf{e}_{1}, \mathbf{e}_{2}, \mathbf{e}_{3}\right)$ which are the reference coordinate system for both $C^{\mathrm{R}}$ and $C^{\mathrm{D}}$. Regarding the notations used for variables, the superscripts 0 and $\mathrm{R}$ correspond to the variables at the initial and co-rotated configurations, respectively. Variables without superscript correspond to the deformed configuration. Subscripts correspond to the starting and ending points of the vector. Over bar '-' denotes a variable in the local coordinate system. Numerical subscripts of the vectors and matrices refer to the node

numbers. For example, in Figure $4, \mathbf{R}_{2}$ is the rotational orientation of the node $2, \mathbf{R}_{d 2}$ is the deformational rotation of the node and $\mathbf{R}_{0}$ is the rigid-body rotation of the node.

A vector $\mathbf{x}$ in the global coordinate system is related to its expression in the local coordinate as follows

$$
\begin{aligned}
& \overline{\mathbf{x}}^{0}=\mathbf{T}_{\mathbf{0}} \mathbf{x}, \\
& \overline{\mathbf{x}}=\mathbf{T} \mathbf{x}
\end{aligned}
$$

where $\mathbf{T}_{\mathbf{0}}$ and $\mathbf{T}$ are the transformation tensors of a location in $C^{0}$ and $C^{\mathrm{D}}$, from the global to local coordinate systems which are expressed as 


$$
\begin{aligned}
& \mathbf{T}_{\mathbf{0}}=\left[\begin{array}{lll}
\mathbf{e}_{1}^{0} & \mathbf{e}_{2}^{0} & \mathbf{e}_{3}^{0}
\end{array}\right]^{T} \\
& \mathbf{T}=\left[\begin{array}{lll}
\mathbf{e}_{1} & \mathbf{e}_{2} & \mathbf{e}_{3}
\end{array}\right]^{T}
\end{aligned}
$$

The displacement of a node $a$ is decomposed into rigid-body and deformational components:

$$
\mathbf{u}_{a}=\mathbf{u}_{r a}+\mathbf{u}_{d a}
$$

where

$$
\begin{aligned}
& \mathbf{u}_{r a}=\mathbf{x}_{a}^{R}-\mathbf{x}_{a}^{0} \\
& \mathbf{u}_{d a}=\mathbf{x}_{a}-\mathbf{x}_{a}^{R}
\end{aligned}
$$

Similarly, the rotation of the node $\mathbf{R}_{a}$ can be decomposed into a rigid-body rotation $\mathbf{R}_{0}$ for nodal rotation in $C^{0} \rightarrow C^{\mathrm{R}}$ and a deformational rotation $\mathbf{R}_{d a}$ as

$$
\mathbf{R}_{a}=\mathbf{R}_{d a} \mathbf{R}_{0}
$$

In the co-rotated local coordinate system of the element, the deformational displacement vector $\overline{\mathbf{p}}_{d}$ including translational and rotational degrees of freedom of the element, $\overline{\mathbf{p}}_{d}=\left\{\overline{\mathbf{u}}_{d}^{T} \overline{\boldsymbol{\theta}}_{d}^{T}\right\}^{T}$, is related to the element internal force $\overline{\mathbf{f}}$ and element stiffness matrix $\overline{\mathbf{K}}$ in a linearized form as follows

$$
\overline{\mathbf{f}}=\overline{\mathbf{K}} \overline{\mathbf{p}}_{d}
$$

Since the problem involves small strains and small deformational degrees of freedom, a linear FE formulation is used in order to form $\overline{\mathbf{f}}$ and $\overline{\mathbf{K}}$ of the element in the local coordinate system for $C^{\mathrm{D}}$ which are typically defined as the following integrals over the volume of the element $\Omega_{e}$ :

$$
\overline{\mathbf{f}}=\int_{\Omega_{e}} \overline{\mathbf{B}}^{T} \overline{\boldsymbol{\sigma}} d V ; \quad \overline{\mathbf{K}}=\int_{\Omega_{e}} \overline{\mathbf{B}}^{T} \overline{\mathbf{D}} \overline{\mathbf{B}} d V
$$

where $\overline{\mathbf{B}}$ is the strain-displacement relations matrix, $\overline{\boldsymbol{\sigma}}$ is the stress, $V$ is the volume and $\overline{\mathbf{D}}$ is the elasticity matrix. 
The element considered in this study is a three-node linear planar element with six degree of freedom per node which is obtained by combining the Discrete Kirchhoff Theory (DKT) plate bending element [36] and optimal membrane element [37], as shown in Figure 5. It is noted that shear locking could occur in bending dominant structures, which could be solved by considering reduced integration or modifying the shape functions of the element. However, this option is not being considered in the present study since our main focus is to highlight the use of nonlinear electro-active materials for inducing three dimensional shape reconfigurations. We avoid shear locking by choosing appropriate geometrical parameters. In the next step of the CRFE, the local quantities and tensors for each element should be transformed to the global coordinate system and also the rigid body motion of each element should be added before assembling and obtaining the total displacement and internal force vectors and stiffness matrix for the entire structure. The routine process of adding of rigid body motions and the CRFE related tensor transformations are given in Appendix A. This approach leads to nonlinear equations for which an incremental and iterative solution is used.

Next, the CRFE formulation is extended for the deformations of smart composite plates when the viscoelastic thin substrate is considered. A general constitutive relation for a linear viscoelastic material is written as:

$$
\boldsymbol{\sigma}(\mathbf{x}, t)=\int_{0}^{t} \mathbf{G}(t-\tau) \frac{\partial \boldsymbol{\varepsilon}(\mathbf{x}, t)}{\partial \tau} d \tau
$$

or in tensorial component form is

$$
\sigma_{i j}(\mathbf{x}, t)=\int_{0}^{t} G_{i j k l}(t-\tau) \frac{\partial \varepsilon_{k l}(\mathbf{x}, t)}{\partial \tau} d \tau
$$

where $\mathbf{G}$ is time-dependent relaxation tensor of the material. As we deal with a plane stress condition two scalar indices are considered, $i, j, k, l=x, y$. In the $\mathrm{FE}$ formulation and incremental iterative process, the incremental quantities are expressed in terms of a discrete interval of time. To obtain small deformational displacements of an element, the incremental form of the viscoelastic constitutive equation is done similar to the procedure performed by Zocher et al [38] and presented here briefly. The incremental stress during time increment $\Delta t$ from $t_{n}$ to $t_{n+1}$ on interval $\left[t_{n}, t_{n+1}\right]$ is written as 


$$
\Delta \boldsymbol{\sigma}=\int_{0}^{t_{n+1}} \mathbf{G}\left(t_{n+1}-\tau\right) \frac{\partial \boldsymbol{\varepsilon}(\mathbf{x}, t)}{\partial \tau} d \tau-\int_{0}^{t_{n}} \mathbf{G}\left(t_{n}-\tau\right) \frac{\partial \boldsymbol{\varepsilon}(\mathbf{x}, t)}{\partial \tau} d \tau
$$

or

$$
\Delta \sigma_{i j}=\int_{t_{n}}^{t_{n+1}} G_{i j k l}\left(t_{n+1}-\tau\right) \frac{\partial \varepsilon_{k l}(\mathbf{x}, \tau)}{\partial \tau} d \tau+\int_{0}^{t_{n}} \Delta G_{i j k l} \frac{\partial \varepsilon_{k l}(\mathbf{x}, \tau)}{\partial \tau} d \tau
$$

The following series of exponential function is chosen for describing the components of the material relaxation tensor:

$$
G_{i j k l}(t)=G_{i j k l_{\infty}}+\sum_{m=1}^{N_{i j k l}} G_{i j k l_{m}} e^{-\frac{t}{\rho_{i j l_{m}}}}
$$

In calculating the integrals in Eq. (27), $\partial \boldsymbol{\varepsilon} / \partial \tau$ is approximated as a constant, $\boldsymbol{\varepsilon}^{\cdot}$, and $\Delta \boldsymbol{\varepsilon}=\boldsymbol{\varepsilon}^{\bullet} \Delta t$ over the interval $\left[t_{n}, t_{n+1}\right]$. Thus, an incremental form of the constitutive Eq. (27) can be written as

$$
\Delta \boldsymbol{\sigma}=\mathbf{G}^{\prime} \boldsymbol{\varepsilon}^{\cdot}+\Delta \boldsymbol{\sigma}^{R}
$$

where

$$
\begin{aligned}
& \mathbf{G}^{\prime}=\int_{t_{n}}^{t_{n+1}} \mathbf{G}\left(t_{n+1}-\tau\right) d \tau, \\
& \Delta \boldsymbol{\sigma}^{R}=\int_{0}^{t_{n}}\left[\mathbf{G}\left(t_{n+1}-\tau\right)-\mathbf{G}\left(t_{n}-\tau\right)\right] \frac{\partial \boldsymbol{\varepsilon}(\mathbf{x}, t)}{\partial \tau} d \tau
\end{aligned}
$$

For FE implementation, the principle virtual work, similar to the elastic problem, for time-dependent deformations at $t+\Delta t$ are

$$
\int_{\Omega^{e}}\left(\boldsymbol{\sigma}^{t}+\Delta \boldsymbol{\sigma}\right)\left(\hat{\boldsymbol{\varepsilon}}^{t}+\Delta \hat{\boldsymbol{\varepsilon}}\right) d V=\int_{\partial \Omega^{e}} \mathbf{t}^{t+\Delta t} \boldsymbol{\psi}^{t+\Delta t} d S+\int_{\Omega^{e}} \rho \mathbf{q}^{t+\Delta t} \boldsymbol{\Psi}^{t+\Delta t} d V
$$

where $\mathbf{t}$ and $\mathbf{q}$ are the surface traction and body force vectors, respectively; $\rho$ is the density of the medium and $\boldsymbol{\psi}$ is a vector of arbitrary admissible displacement functions and each component of $\hat{\boldsymbol{\varepsilon}}$ is defined in terms of components of $\boldsymbol{\psi}$ as $\hat{\varepsilon}_{i j}=0.5\left(\psi_{i, j}+\psi_{j, i}\right)$.

By considering the equilibrium condition at the previous time $t$ and substituting Eq. (30) into the equation, Eq. (33), the equilibrium equation at current time is 


$$
\int_{\Omega^{e}} \Delta \hat{\mathbf{\varepsilon}} \mathbf{G}^{\prime} \Delta \boldsymbol{\varepsilon} d V=\int_{\partial \Omega^{e}} \mathbf{t}^{t+\Delta t} \Delta \boldsymbol{\psi} d S+\int_{\Omega^{e}} \rho \mathbf{q}^{t+\Delta t} \Delta \boldsymbol{\psi} d V-\int_{\Omega^{e}} \Delta \hat{\mathbf{\varepsilon}} \boldsymbol{\sigma}^{t} d V-\int_{\Omega^{e}} \Delta \hat{\mathbf{\varepsilon}} \Delta \boldsymbol{\sigma}^{R} d V
$$

The incremental strain is given as

$$
\Delta \boldsymbol{\varepsilon}=\mathbf{B} \Delta \mathbf{u}, \Delta \hat{\boldsymbol{\varepsilon}}=\mathbf{B} \Delta \psi
$$

where $\mathbf{u}$ is the displacement of the element,

$$
\Delta \mathbf{u}=\mathbf{N} \Delta \mathbf{U}_{n}, \Delta \boldsymbol{\psi}=\mathbf{N} \Delta \boldsymbol{\Psi}_{n}
$$

where $\mathbf{U}_{n}$ and $\Psi_{n}$ are the nodal displacement vectors of the element and $\mathbf{N}$ is the shape function matrix. By replacing Eqs. (34) and (35) and rearranging, the Eq. (33) is written as

$$
\begin{aligned}
& \int_{\Omega_{e}}\left(\mathbf{B} \Delta \boldsymbol{\Psi}_{n}\right)^{T} \mathbf{G}^{\prime} \mathbf{B} \Delta \mathbf{U}_{n} d V=\int_{\Omega^{e}}\left(\mathbf{B} \Delta \boldsymbol{\Psi}_{n}\right)^{T} \boldsymbol{\sigma}^{t} d V+\int_{\Omega^{e}}\left(\mathbf{B} \Delta \boldsymbol{\Psi}_{n}\right)^{T} \Delta \boldsymbol{\sigma}^{R} d V \\
& +\int_{\partial \Omega^{e}}\left(\mathbf{N} \Delta \boldsymbol{\Psi}_{n}\right)^{T} \mathbf{t}^{t+\Delta t} d S+\int_{\Omega^{e}}\left(\mathbf{N} \Delta \boldsymbol{\Psi}_{n}\right)^{T} \rho \mathbf{q}^{t+\Delta t} d V
\end{aligned}
$$

It is noted that $\Delta \boldsymbol{\Psi}_{n}$ is arbitrary, it is simplified as $\mathbf{K} \Delta \mathbf{U}_{n}=\mathbf{F}$ for the element. In the CRFE approach, this gives the force and stiffness of the element in a co-rotated configuration $C^{\mathrm{R}}$. The aforementioned CRFE formulations are then used in the same way as for the elastic case in order to obtain the structural stiffness matrix and force vector.

The Newton-Raphson iterative algorithm with load control is adopted to determine the displacement solution of the structure under applied loads. Briefly, due to nonlinearity of the problem, the solution obtained by an increment-iterative process is as follows: Consider the equilibrium point of the structure after $n$ load steps with known equilibrated displacement solution $\mathbf{U}^{(n)}$, structural tangent stiffness $\mathbf{K}_{S t}^{(n)}$ and internal force $\mathbf{F}_{S t}^{(n)}$ in the first iteration of $(n+1)^{\text {th }}$ increment, the following equation is considered as the predictor:

$$
\delta \mathbf{U}_{(1)}^{(n+1)}=\left(\mathbf{K}_{S t(1)}^{(n+1)}\right)^{-1}\left(\lambda^{(n+1)} \mathbf{F}_{\text {ext }}\right)
$$

where $\delta \mathbf{U}_{(1)}^{(n+1)}$ is the initial guess of the structure's incremental displacement by using $\mathbf{K}_{S t}^{(n)}$ at the end of $n^{\text {th }}$ increment as the trial for tangent stiffness $\mathbf{K}_{S t}^{(n+1)}$ due to the $(n+1)^{\text {th }}$ incremental load $\lambda^{(n+1)} \mathbf{F}_{\text {ext }} ; \mathbf{F}_{\text {ext }}$ is the total external load applied to the structure and $\lambda^{(n+1)}$ is the load factor of the 
$(n+1)^{\text {th }}$ load step. The subscripts and superscripts in the parentheses stand for the iteration number and increment number, respectively.

The solution is updated as $\mathbf{U}^{(n+1)}=\mathbf{U}^{(n)}+\delta \mathbf{U}_{(1)}^{(n+1)}$ and then, the tangent stiffness and internal force of the structure is updated. Starting from the $2^{\text {nd }}$ iteration, the following equation is solved for each iteration $(i)$

$$
\delta \mathbf{U}_{(i)}^{(n+1)}=\left(\mathbf{K}_{S t(i)}^{(n+1)}\right)^{-1}\left(\lambda^{(n+1)} \mathbf{F}_{e x t}-\mathbf{F}_{S t(i)}\right)
$$

and as for the first iteration, $\mathbf{U}^{(n+1)}$ is updated and $\mathbf{K}_{S t}^{(n+1)}$ and $\mathbf{F}_{S t}$ are calculated for the next iteration. This process is repeated until a convergence criterion is met. The following convergence criterion is considered

$$
\frac{\left\|\delta \mathbf{U}_{(i+1)}^{(n+1)}\right\|}{\left\|\mathbf{U}^{(n+1)}\right\|} \leq 10^{-3}
$$

To improve the computational efficiency, the load control strategy is adopted in which the magnitude of the load increment is adjusted depending on convergence rate in previous step: a maximum allowable iteration $i_{\max }$ is chosen for a single load step; For the $n^{\text {th }}$ load step, if convergence occurs after $j$ iterations, for the $\mathrm{n}+1$ th step, the magnitude of load factor $\lambda^{(n)}$ is adjusted as follows

$$
\lambda^{(n+1)}=\left(\frac{i_{\max }}{j}\right)^{2} \lambda^{(n)}
$$

For example, if at the $n^{\text {th }}$ load step, convergence occurs after smaller number iterations than $i_{\max }$, the load factor is increased for the next load step.

\section{Boundary value problems}

First, the CRFE solution for electro-active composite plates is reduced to analyze responses of a homogeneous elastic beam. This is done in order to compare the numerical responses with the analytical solution based on Reissner's theory [39] for a cantilever beam under a bending moment equal to $1.5 \mathrm{KN}-\mathrm{m}$ along its free edge, as shown in Figure 6. The 
elastic modulus and Poisson ratio of the beam are taken as $10 \mathrm{MPa}$ and 0.1 respectively. It is seen that the CRFE solution is in an excellent agreement with the analytical solution, implying the accuracy of the numerical approach.

In next examples, deformation analysis of smart composite structures with multiple pairs of active piezoelectric patches is performed. The materials properties of the elastic patch and substrate are presented in Table 3. For all boundary value problems, convergence study is preformed to evaluate the size of elements needed for discretizing the domain for obtaining accurate numerical results.

\subsection{Smart elastic plates}

Figure 7 shows the configuration of a composite plate with one pair of patches stimulated by electric field with various magnitudes when a nonlinear behavior is considered for the piezoelectric material. The green-color region shows the part of plate covered by the patch. One can see the effect of magnitude of electric stimulus on the deformations of the plate. The amount of applied electric field is kept lower than the coercive strength of the piezoelectric material, which is higher than $1.44 \mathrm{MV} / \mathrm{m}$ for PZT G-1195 [40]. This reconfiguration under different magnitudes of stimulus can resemble morphing shape change of wing structures.

In figures $8 \mathrm{a}$ and $\mathrm{b}$ respectively, linear and nonlinear electro-mechanical responses of piezoelectric material as actuators are compared. Under the same magnitude of electric field, $E^{e}$ $=1.3 \mathrm{MV} / \mathrm{m}$, there is a significant effect of the nonlinear electro-mechanical behavior of the actuators on deformed configuration of the structure. Figures 9 shows the corresponding axial strain distributions along the longitudinal direction of the plate corresponding to the deformed plate in figure $8 \mathrm{~b}$. As seen, the strain magnitude over the covered areas with patches is relatively small for the plate that experiences large rotation which shows the possibility of using piezoelectric materials that is limited in small strain to achieve large rotation in structures.

\subsection{Smart viscoelastic plates}

In this section, several boundary value problems are presented to examine the deformations of smart plates comprising of viscoelastic homogeneous substrates and 
piezoelectric actuators. Both elastic piezoceramic patches and viscoelastic AFCs subjected to electric stimuli are considered. The relaxation function of the isotropic substrate is expressed as

$$
E_{p l}(t)=G(t)=0.4+1.6 e^{-t} \mathrm{GPa}
$$

The Poisson ratio of the viscoelastic substrate is assumed time-independent and the same as for elastic substrate, given in Table 3. The materials properties of the piezoelectric and AFC patches and thickness of the components are the same as before. Again, a nonlinear response of piezoelectric material in terms of electric field is taken into account.

The electric field stimulus is modeled with a time dependent function defined in Eq. (42) which has a continuous time derivative in the interval $[0 t]$ so that the Laplace transformation can be used during the considered interval for solving the governing interval equations.

$$
E^{e}(t)=E_{m}^{e}\left(1-e^{-t / t_{r}}\right)
$$

A viscoelastic plate with one pair of piezoceramics patches is subjected to timedependent electric field as described in Eq. (42) with $E_{m}^{e}=1.1 \mathrm{MV} / \mathrm{m}$ and $t_{r}=0.02 \mathrm{sec}$ for total actuation time $T$. Thus, the load almost reaches to $E_{m}^{e}$ and remains constant in less than $0.1 \mathrm{sec}$. The configuration of the plate for different times is shown in figure 10. As seen, the curvature of the plate increases gradually at early time but it almost remains unchanged after $T=0.5 \mathrm{sec}$ and so the configuration of the structure remains constant although the relaxation modulus of the viscoelastic plate decreases over time, Eq. (41). The reason is that the during stress relaxation within the substrate, interaction between the substrate and the patch also decreases under constant stimulus and because the induced moment from the activated patch also depends to the relaxation function thus, the induced moment from the activated patch to the substrate decreases which makes up for rigidity reduction of the structure and so the shape remains constant. Figure 11 shows the induced moments to the substrate under the aforementioned electric stimulus, Eq. (41). Since the electric stimulus in the beginning reaches from zero almost to its maximum, the induced moment increases at first and later it decreases with time because the stimulus remains almost constant and the relaxation function decreases. 
Figure 12 shows numerical simulation of the lateral deformations of a wing concept for micro UAVs (Figure 12a) by considering piezoelectric actuators and viscoelastic substrate. Similar to previous case, it is seen that deformation of the structure increases fast for $0<T<0.05 \mathrm{sec}$ due to an increase of applied electric field but then remains almost constant for $T>0.05 \mathrm{sec}$. The fact that under a constant electric stimulus input prescribed to the elastic piezoelectric actuators, the configuration of the viscoelastic structure is almost time-independent or stable over time is important in practice. This enables engineers to use polymers in design of for example lightweight structures with desired shape changes under certain magnitudes of stimulus without being concerned about time effect on their configurations.

In the following examples, we consider the actuators made of AFCs with viscoelastic effects. A slender viscoelastic plate with one pair of AFC patches is subjected to time-dependent electric field as described in Eq. (42) with $E_{m}^{e}=0.5 \mathrm{MV} / \mathrm{m}$ and $t_{r}=0.02 \mathrm{sec}$. The configurations of the plate for different times are shown in Figure 13. As seen, the curvature of the plate increases over time but its rate of increase reduces gradually. For large enough $T$, the configuration of the structure almost does not change, which is at the relaxed conditions for both the actuator and substrate. Unlike the plate with the elastic piezoelectric-ceramics patches, the effect of the viscoelastic AFC patches on the deformation is observed. In this case the significant time-dependent effect is due to the piezoelectric coefficient of the AFC that change with time (Eq. (13)). The piezoelectric effect of the AFC leads to an increase of the induced moment to the substrate over time. As time increases, reduction of the rate of the piezoelectric effect is seen until it becomes almost constant and thus, the deformation of the structure does not increase noticeably after some time.

In Figure 14, another example of a viscoelastic plate actuated by four active pairs of AFC patches with $E_{m}^{e}=0.5 \mathrm{MV} / \mathrm{m}$ is shown. Over time, the four arms along four edges of the squareshape base fold gradually which can resemble a self-folding actuated structure.

Finally, in the following two examples, twisting configurations of two smart viscoelastic plate after $T=0.1 \mathrm{sec}$ and $1 \mathrm{sec}$ with two pairs of quadrilateral-shape patches stimulated in the same directions (Figure 15) and opposite directions (Figure 16) are shown from different view angles. It is seen that different arrangements of AFC patches can induce various threedimensional shape changes in the originally planar structures. 


\section{Conclusion}

Deformation analyses of smart flexible and foldable electro-active composites have been performed in this study. The composite structures comprise of thin planar substrates integrated with piezoelectric actuators perfectly bonded to the top and bottom surfaces of the substrates. The actuators considered here are piezoelectric ceramics, which is elastic, and AFC, which is viscoelastic. Nonlinear electro-mechanical responses are considered for both piezoelectric ceramics and AFC since for actuating purposes relatively large electric field is often prescribed. Viscoelastic behaviors are considered for the polymeric substrates. By prescribing electric field inputs to the actuators the planar composite structures can be formed into three-dimensional shapes predominantly by rotations while the in-plane strains and stretch remain small. The CRFE method has been adopted for the large deformation analyses of the electro-active composite structures.

Several arrangements of piezoelectric patches and different history of electric field inputs have been considered in order to obtain various shape changes in the planar structures. The effects of viscoelastic substrates and nonlinear electro-mechanical coupling properties on the deformed shapes have been examined. It is concluded that when elastic piezoelectric patches, such as piezoelectric ceramics, are bonded to viscoelastic substrates the time-dependent deformations in the overall structures are negligible since the resulting bending moments required to induce rotations to the structures decrease simultaneously with stress relaxation in the viscoelastic substrates. However, significant time-dependent deformations are observed when the viscoelastic AFC patches are considered, in which the structures continue to experience shape changes even after the electric field input is completely prescribed. The nonlinear electromechanical coupling behaviors of the actuators significantly influence the deformed shapes of the structures. The presented methods and examples of boundary value problems discussed in this study can help designers in simulating desired deformed shapes and determining placements of piezoelectric patches external stimuli to be prescribed prior to fabricating smart and flexible composites. The analyses can also help in selecting materials for both substrates and actuators during the design of foldable and flexible composite structures. 


\section{Acknowledgement}

This research is sponsored by the Air Force Office of Scientific Research (AFOSR) under grant FA9550-14-1-0234 and the National Science Foundation (NSF) under grant CMMI-1437086.

\section{References}

[1] Srinivasa, A. R., On a class of Gibbs potential-based nonlinear elastic models with small strain. Acta Mechanica, 2015. 226: p. 571-583.

[2] P. M. Naghdi and L. Vongsarnpigoon, $A$ theory of shells with small strain accompanied by moderate rotation. Archive for Rational Mechanics and Analysis, 1983. 83: p. 245-283.

[3] A. A. Bent and N. W. Hagood, Piezoelectric fiber composites with interdigitated electrodes. Journal of Intelligent Material Systems and Structures, 1997. 8(11): p. 903-919.

[4] C. P. Wu, K. H. Chiu and Y. M. Wang, a review on the three dimensional analytical approaches of multilayered and functionally graded piezoelectric plates and shells. CMC: Computers, Materials \& Continua, 2008. 8: p. 93-132.

[5] L. Kang, Q. L. Zhang and Q. L. Wang, Linear and geometrically nonlinear analysis of novel flat shell elements with rotational degrees of freedom Finite Elements in Analysis and Design, 2009. 45: p. 386-392.

[6] E. Gal and R. levy, Geometrically nonlinear analysis of shell structures using a flat triangular shell finite element. Archives of Computational Methods in Engineering, 2006. 13: p. 331-388.

[7] S. Timoshenko and S. Woinowsky-Krieger, Theory of plates and shells. 1959: McGraw-Hill.

[8] Ch.-F. Chen and J.-H. Chen, Nonlinear study of large deflection of simply supported piezoelectric layeredplate under initial tension. International Journal of Mechanical Sciences, 2011. 53: p. 485-493.

[9] C.X. Xue, E. Pan, S.Y. Zhang and H.J. Chu, Large deflection of a rectangular magnetoelectroelastic thin plate. Mechanics Research Communications, 2011. 38: p. 518-523.

[10] G.A. Wempner, Finite elements, finite rotations and small strains of flexible shells. International journal of solid structures, 1969. 5: p. 117-153.

[11] T. Belytschko and B. J. Hsieh, Non -linear transient finite element analysis with convected co-ordinates. International journal of numerical methods in engineering, 1973. 7: p. 255-271.

[12] B . Nour-Omid and C . C. Rankin Finite rotation analysis and consistent linearization using projectors. Computational methods in applied mechanics, 1991. 93: p. 353-384.

[13] Cardona, A., An integrated approach to mechanism analysis, . 1989, University of Liege Belgium.

[14] M. A. Crisfield, A consistent corotational formulation for nonlinear three-dimensional beam element. Computational methods in applied mechanics, 1990. 81: p. 131-150.

[15] M. A. Crisfield, Nonlinear Finite Element Analysis of Solids and Structures, Vol. 2, Advanced Topics. 1997, Chichester: Wiley.

[16] M. A. Crisfield and G. F. Moita, A unified co-rotational for solids, shells and beams. International journal of solid structures, 1996. 33: p. 2969-2992.

[17] C.A. Felippa and B.A. Hagen, A unified formulation of small-strain corotational finite elements: I. Theory. Computational methods in applied mechanics and Engineering, 2005. 194: p. 2285-2335.

[18] F.S. Almeida and A.M. Awrunch, Corotational nonlinear dynamic analysis of laminated composite shells. Finite Elements Analysis Design, 2011. 47: p. 1131-1145.

[19] X.M. An and M. Xu, An improved geometrically nonlinear algorithm and its application for nonlinear aeroelasticity. Chinese journal of theoretical applied mechanics, 2011. 4314: p. 97-104. 
[20] Y. JinSong and X. PinQi, Finte element corotational formulation for geometric nonlinear analysis of thin shells with large rotation and small strain. Science China, 2012. 55: p. 3142-3152.

[21] Y. C. Cai and S. N. Atluri, Large rotation analyses of plate/shell structures based on the primal variational principle and a fully nonlinear theory in the updated Lagrangian co-rotational reference frame. CMES: Computer modeling in engineering and sciences, 2012. 83: p. 249-273.

[22] Y. C. Cai, J. K. P., S. N. Atluri, A triangular plate element with drilling degrees of freedom, for large rotation analyses of built-up plate/shell structures, based on the Reissner variational principle and the von Karman nonlinear theory in the co-rotational reference frame. CMES: Computer modeling in engineering and sciences, 2010. 61: p. 273-312.

[23] H.F. Tiersten, Electroelastic equations for electroded thin plates subject to large driving voltages Journal of Applied Physics 1993. 74(5): p. 3389 - 3393.

[24] V. Tajeddini, Ch.-H. Lin, A. Muliana and M. Lévesque, Average electro-mechanical properties and responses of active composites. Computational Materials Science, 2014. 82: p. 405-414.

[25] P. Tan and L. Tong, Micro-electromechanics models for piezoelectric-fiber-reinforced composite materials. Composites Science and Technology, 2001. 61(5): p. 759-769.

[26] A. Muliana and Ch.-H. Lin, A Multi-scale Formulation for Predicting Non-linear Thermo-electromechanical Response in Heterogeneous Bodies. Journal of Intelligent Material Systems and Structures, 2011. 22: p. 723-738.

[27] Mollayousef, A. S., Nonlinear and rate dependent hysteresis electromechanical responses of ferro electric materials. 2015, Texas A\&M University.

[28] H. B. Atitallah, Characterization and modeling of active fiber composites. 2014, Pennsylvania State University.

[29] H. B. Atitallah, Z. Ounaies and A. H. Muliana, Temperature and time dependence of the electromechanical properties of flexible active fiber composites. Smart Materials and Structures, 2016.

[30] V. Tajeddini, H. B. Atitallah, A. Muliana and Z. Ounaies, Nonlinear Viscoelastic Behavior of Active Fiber Composites. Journal of Engineering Materials and Technology, 2014. 136(2).

[31] E. K. Dimitriadis, C. R. Fuller and C. A. Rogers, Piezoelectric actuators for distributed vibration excitation of thin plates. Journal of Vibrations and Acoustics 1991. 113: p. 100-107.

[32] K. A. Khan, A. H. Muliana, H. B. Atitallah and Z. Ounaies, Time-dependent and energy dissipation effects on the electro-mechanical response of PZTs. Mechanics of Materials, 102, pp. 74-89, 2016.

[33] Y. C. Fung, Biomechanics: Mechancial properties of living tissues. 1981, New York: Springe.

[34] H. B. Atitallah, Z. Ounaies and A. Muliana, A parametric study on flexible electro-active composites: Importance of geometry and matrix properties. Journal of Intelligent Material Systems and Structures, 2014.

[35] C. A. Felippa and B. A. Hagen, A unified formulation of small-strain corotational finite elements: I. Theory. Computational methods in applied mechanics and Engineering, 2005. 194: p. 2285-2335.

[36] J.L. Batoz, K.J. Bathe and L.W. Ho, A study of three-node triangular plate bending elements. International journal of numerical methods in engineering, 1980. 15: p. 1771-1812.

[37] C.A. Felippa, A study of optimal membrane triangles with drilling freedoms. Computational methods in applied mechanics and Engineering, 2003. 192: p. 2125-2168.

[38] M. A. Zocher and E. Groves, A three-dimensional finite element formulation for thermoviscoelastic orthotropic media. International Journal for Numerical Methods in Engineering, 1997. 40: p. 22672288.

[39] V. Tajeddini and A. Muliana, Nonlinear deformations of piezoelectric composite beams. Composite Structures, 2015. 132: p. 1085-1093.

[40] J. Yang, Special Topics in the Theory of Piezoelectricity. 2009: Springer. 


\section{Appendix A}

This appendix summarizes the formulation in CRFE method [20, 35]. In CR formulation, in addition to pseudo-vector $\boldsymbol{\theta}_{a}$ for the rotational degrees of freedom of node $a$, rotation vector $\boldsymbol{\omega}_{a}$ is utilized which denotes an instantaneous rotation axis about which the rotational angle $\theta$ is measured. For an iterative incremental solution, the incremental deformational rotation $\delta \overline{\boldsymbol{\omega}}_{d a}$ is related to $\delta \overline{\boldsymbol{\theta}}_{d a}$ as follows

$$
\delta \overline{\boldsymbol{\theta}}_{d a}=\mathbf{H}\left(\overline{\boldsymbol{\theta}}_{d a}\right) \delta \overline{\boldsymbol{\omega}}_{d a}
$$

where

$$
\mathbf{H}\left(\overline{\boldsymbol{\theta}}_{d a}\right)=\frac{\partial \overline{\boldsymbol{\theta}}_{d a}}{\partial \overline{\boldsymbol{\omega}}_{d a}}=\mathbf{I}_{3}-\frac{1}{2} \operatorname{spin}\left(\overline{\boldsymbol{\theta}}_{d a}\right)+\eta \operatorname{spin}\left(\overline{\boldsymbol{\theta}}_{d a}\right)^{2}
$$

$\mathbf{I}_{3}$ is $3 \times 3$ identity matrix,

$$
\operatorname{spin}\left(\overline{\boldsymbol{\theta}}_{d a}\right)=\left(\begin{array}{ccc}
0 & -\bar{\theta}_{d a z} & \bar{\theta}_{d a y} \\
\bar{\theta}_{d a z} & 0 & -\bar{\theta}_{d a x} \\
-\bar{\theta}_{d a y} & \bar{\theta}_{d a x} & 0
\end{array}\right)
$$

and

$$
\eta=\frac{1-\frac{1}{2} \bar{\theta}_{d a} \cot \left(\frac{1}{2} \bar{\theta}_{d a}\right)}{\bar{\theta}_{d a}^{2}}
$$

To ensure numerical stability, for small $\bar{\theta}_{d a}$, say $\bar{\theta}_{d a} \leq 3^{\circ}$, a truncated Taylor series of $\eta$ is used:

$$
\eta=\frac{1}{12}+\frac{1}{720} \bar{\theta}_{d a}{ }^{2}+\frac{1}{30240} \bar{\theta}_{d a}^{4}+\frac{1}{1209600} \bar{\theta}_{d a}{ }^{6}+\ldots
$$

The increment of the deformational displacement vector is expressed by $\delta \mathbf{d}_{d}=\left\{\delta \mathbf{u}_{d}^{T} \delta \boldsymbol{\omega}_{d}^{T}\right\}^{T}$ which according to Eq. (A1) relates to $\delta \overline{\mathbf{p}}_{d}=\left\{\delta \overline{\mathbf{u}}_{d}^{T} \delta \overline{\boldsymbol{\theta}}_{d}^{T}\right\}^{T}$ as

$$
\delta \overline{\mathbf{p}}_{d}=\mathbf{H} \delta \overline{\mathbf{d}}_{d}
$$


where

$$
\overline{\mathbf{H}}=\operatorname{diag}\left[\begin{array}{llllll}
\mathbf{I}_{3} & \overline{\mathbf{H}}_{1} & \mathbf{I}_{3} & \overline{\mathbf{H}}_{2} & \mathbf{I}_{3} & \overline{\mathbf{H}}_{3}
\end{array}\right]
$$

consists of submatrices $\mathbf{I}_{3}$ and nodal submatrices $\overline{\mathbf{H}}_{a}=\overline{\mathbf{H}}\left(\overline{\boldsymbol{\theta}}_{d a}\right)(a=1,2,3)$.

One of the main auxiliary matrices in the CR formulation is called projector matrix $\overline{\mathbf{P}}$ which is formed to extract the deformational parts of the translations and rotations from the total translations and rotations of the element:

$$
\delta \overline{\mathbf{d}}_{d}=\overline{\mathbf{P}} \delta \overline{\mathbf{d}}
$$

The derivation of $\overline{\mathbf{P}}$ is explained in details in $[17,18]$ and is not repeated here. A submatrix of the projector matrix, $\overline{\mathbf{P}}_{a b}$ is expressed as follows

$$
\overline{\mathbf{P}}_{a b}=\left(\begin{array}{cc}
\mathbf{U}_{a b}+\overline{\mathbf{S}}_{a} \overline{\mathbf{G}}_{u, b} & 0_{3} \\
-\overline{\mathbf{G}}_{u, b} & \delta_{a b} \mathbf{I}_{3}
\end{array}\right) ; \quad(a, b=1,2,3)
$$

where

$$
\mathbf{U}_{a b}=\left(\delta_{a b}-\frac{1}{3}\right) \mathbf{I}_{3}, \overline{\mathbf{S}}_{a}=\operatorname{spin}\left(\overline{\mathbf{x}}_{a}\right)=\left(\begin{array}{ccc}
0 & -\bar{x}_{a z} & \bar{x}_{a y} \\
\bar{x}_{a z} & 0 & -\bar{x}_{a x} \\
-\bar{x}_{a y} & \bar{x}_{a x} & 0
\end{array}\right)
$$

and $\overline{\mathbf{G}}_{u, b}$ is the gradient of incremental rigid-body rotation of the element with respect to the nodal displacement vector for node $b$ in the local coordinate system. At each node, $\overline{\mathbf{G}}_{u, b}$ is defined as

$$
\overline{\mathbf{G}}_{u, 1}=\frac{1}{2 A}\left(\begin{array}{ccc}
0 & 0 & \bar{x}_{23} \\
0 & 0 & \bar{y}_{23} \\
0 & -2 A / l_{12} & 0
\end{array}\right), \overline{\mathbf{G}}_{u, 2}=\frac{1}{2 A}\left(\begin{array}{ccc}
0 & 0 & \bar{x}_{13} \\
0 & 0 & \bar{y}_{13} \\
0 & -2 A / l_{12} & 0
\end{array}\right), \overline{\mathbf{G}}_{u, 3}=\frac{1}{2 A}\left(\begin{array}{ccc}
0 & 0 & \bar{x}_{21} \\
0 & 0 & \bar{y}_{21} \\
0 & 0 & 0
\end{array}\right)
$$

where $A$ is area of the element and $\bar{x}_{i j}=\bar{x}_{i}-\bar{x}_{j}, \bar{y}_{i j}=\bar{y}_{i}-\bar{y}_{j}(i, j=1,2,3)$ in which $\bar{x}$ and $\bar{y}$ are the local coordinates of the element nodes.

Having local internal force vector of the element $\overline{\mathbf{f}}$ from linear FE formulation, because of virtual work invariance under rigid body motion, $\delta \mathbf{d}^{T} \mathbf{f}=\delta \overline{\mathbf{p}}_{d}^{T} \overline{\mathbf{f}}$, one can obtain internal force 
vector of an element in global coordinate system, $\mathbf{f}$, by using projector and transformation tensors as follows

$$
\mathbf{f}=\mathbf{T}_{e l}^{T} \overline{\mathbf{P}}^{T} \overline{\mathbf{H}}^{T} \overline{\mathbf{f}}
$$

where $\mathbf{T}_{e l}$ is composed of the transformation submatrices $\mathbf{T}$ given in Eq. (20) as follows:

$$
\mathbf{T}_{e l}=\operatorname{diag}[\mathbf{T} \mathbf{T} \mathbf{T} \text { T } \mathbf{T} \text { T }]
$$

Taking the variation of $\mathbf{f}$ results in the consistent tangent matrix of the element:

$$
\begin{aligned}
\delta \mathbf{f} & =\delta \mathbf{T}_{e l}^{T} \overline{\mathbf{P}}^{T} \overline{\mathbf{H}}^{T} \overline{\mathbf{f}}+\mathbf{T}_{e l}^{T} \delta \overline{\mathbf{P}}^{T} \overline{\mathbf{H}}^{T} \overline{\mathbf{f}}+\mathbf{T}_{e l}^{T} \overline{\mathbf{P}}^{T} \delta \overline{\mathbf{H}}^{T} \overline{\mathbf{f}}+\mathbf{T}_{e l}^{T} \overline{\mathbf{P}}^{T} \overline{\mathbf{H}}^{T} \delta \overline{\mathbf{f}} \\
& =\left(\mathbf{K}_{G R}+\mathbf{K}_{G P}+\mathbf{K}_{G M}+\mathbf{K}_{M}\right) \delta \mathbf{d}=\mathbf{K}_{T} \delta \mathbf{d}
\end{aligned}
$$

As seen in the equation above, the tangent stiffness of the element in the global coordinate system includes four terms. The first three terms, $\mathbf{K}_{G R}+\mathbf{K}_{G P}+\mathbf{K}_{G M}$, are the components of geometric stiffness, $\mathbf{K}_{G}$, and the fourth term $\mathbf{K}_{M}$ is known as material stiffness. $\mathbf{K}_{G R}$ is the rotational geometric stiffness expressed as

$$
\mathbf{K}_{G R}=-\mathbf{T}_{e l}^{T} \overline{\mathbf{F}}_{n m} \overline{\mathbf{G}} \mathbf{T}_{e l}
$$

where

$$
\begin{aligned}
& \overline{\mathbf{G}}=\left[\begin{array}{llllll}
\overline{\mathbf{G}}_{u, 1} & \mathbf{0}_{3} & \overline{\mathbf{G}}_{u, 2} & \mathbf{0}_{3} & \overline{\mathbf{G}}_{u, 3} & \mathbf{0}_{3}
\end{array}\right], \\
& \overline{\mathbf{F}}_{n m}=\left[\operatorname{spin}\left(\overline{\mathbf{n}}_{p, 1}\right)^{T} \operatorname{spin}\left(\overline{\mathbf{m}}_{p, 1}\right)^{T} \ldots \operatorname{spin}\left(\overline{\mathbf{n}}_{p, 3}\right)^{T} \operatorname{spin}\left(\overline{\mathbf{n}}_{p, 3}\right)^{T}\right]^{T}
\end{aligned}
$$

where $\overline{\mathbf{n}}_{p, a}$ and $\overline{\mathbf{m}}_{p, a}$ are the force and moment components of vector $\overline{\mathbf{f}}_{p}=\overline{\mathbf{P}}^{T} \overline{\mathbf{H}}^{T} \overline{\mathbf{f}} . K_{G P}$ is the equilibrium projection geometric stiffness expressed as

$$
\mathbf{K}_{G P}=-\mathbf{T}_{e l}^{T} \overline{\mathbf{G}}^{T} \overline{\mathbf{F}}_{n}^{T} \overline{\mathbf{P}} \mathbf{T}_{e l}
$$


where

$$
\overline{\mathbf{F}}_{n}=\left[\operatorname{spin}\left(\overline{\mathbf{n}}_{p, 1}\right)^{T} \mathbf{0}_{3} \operatorname{spin}\left(\overline{\mathbf{n}}_{p, 2}\right)^{T} \mathbf{0}_{3} \operatorname{spin}\left(\overline{\mathbf{n}}_{p, 3}\right)^{T} \mathbf{0}_{3}\right]^{T}
$$

$\mathbf{K}_{G M}$ is the moment-correction geometric stiffness due to variation of $\mathbf{H}$ and expressed as

$$
\mathbf{K}_{G P}=\mathbf{T}_{e l}^{T} \overline{\mathbf{P}}^{T} \overline{\mathbf{L}} \overline{\mathbf{P}} \mathbf{T}_{e l}
$$

where

$$
\mathbf{L}=\operatorname{diag}\left[\begin{array}{llllll}
\mathbf{0}_{3} & \overline{\mathbf{L}}_{1} & \mathbf{0}_{3} & \overline{\mathbf{L}}_{2} & \mathbf{0}_{3} & \overline{\mathbf{L}}_{3}
\end{array}\right]^{T}
$$

where the nodal submatrix $\bar{L}_{a}$ is

$$
\overline{\mathbf{L}}_{a}=\left\{\eta\left(\overline{\boldsymbol{\theta}}_{d a} \overline{\mathbf{m}}_{a} \mathbf{I}_{3}+\overline{\boldsymbol{\theta}}_{d a} \overline{\mathbf{m}}_{a}^{T}-2 \overline{\mathbf{m}}_{a} \overline{\boldsymbol{\theta}}_{d a}^{T}\right)+\mu \operatorname{spin}\left(\overline{\boldsymbol{\theta}}_{d a}\right)^{2} \overline{\mathbf{m}}_{a} \overline{\boldsymbol{\theta}}_{d a}^{T}-\frac{1}{2} \operatorname{spin}\left(\mathbf{m}_{a}\right)\right\} \overline{\mathbf{H}}\left(\overline{\boldsymbol{\theta}}_{d a}\right)
$$

$\eta$ is defined already in Eq.(30) and $\mu=d \eta / d \bar{\theta}_{d a} / \bar{\theta}_{d a}$.

The material stiffness matrix is obtained by congruential transformation of the local stiffness $\overline{\mathbf{K}}$ to the global frame:

$$
\mathbf{K}_{M}=\mathbf{T}_{e l}{ }^{T} \overline{\mathbf{P}}^{T} \overline{\mathbf{H}}^{T} \overline{\mathbf{K}} \overline{\mathbf{H}} \overline{\mathbf{P}} \mathbf{T}_{e l}
$$

Finally, the following expression gives the tangent stiffness of the element in the global coordinate system:

$$
\mathbf{K}_{T}=\mathbf{T}_{e l}^{T}\left(\overline{\mathbf{P}}^{T} \overline{\mathbf{H}}^{T} \overline{\mathbf{K}} \overline{\mathbf{H}} \overline{\mathbf{P}}+\overline{\mathbf{P}}^{T} \overline{\mathbf{L}} \overline{\mathbf{P}}-\overline{\mathbf{G}}^{T} \overline{\mathbf{F}}_{n}^{T} \overline{\mathbf{P}}-\overline{\mathbf{F}}_{n m} \overline{\mathbf{G}}\right) \mathbf{T}_{e l}
$$

Once the internal force and tangent stiffness for each element are defined, the structural stiffness $\mathbf{K}_{S t}$ and internal force $\mathbf{F}_{S t}$ are formed for the entire structure. 


\section{Tables}

Table 1. Normalized parameters of the relaxation function

\begin{tabular}{|c|c|c|}
\hline$n$ & $\Upsilon_{n}$ & $\tau_{n}(\min )$ \\
\hline$\infty$ & 0.3934 & - \\
\hline 1 & 0.1449 & 1.04976 \\
\hline 2 & 0.00277 & 0.00923 \\
\hline 3 & 0.00069 & 49.75124 \\
\hline 4 & 0.1771 & 3.32799 \\
\hline 5 & 0.1078 & 0.01006 \\
\hline 6 & 0.1759 & 56.17978 \\
\hline
\end{tabular}

Table 2. Parameters of the Prony series for piezoelectric coefficient

\begin{tabular}{|c|c|c|}
\hline$n$ & $d_{p x x, n}(\mathrm{pm} / \mathrm{V})$ & $\lambda_{n}(\min )$ \\
\hline 0 & 110 & - \\
\hline 1 & 10 & 0.2 \\
\hline 2 & 120 & 1 \\
\hline 3 & 10 & 10 \\
\hline 4 & 140 & 60 \\
\hline
\end{tabular}

Table 3. Properties of the components of the smart plate

\begin{tabular}{|c|c|}
\hline Patch (PZT G-1195) [23] & Substrate \\
\hline$d_{31}=d_{32}=180 \mathrm{pm} / \mathrm{V}, \quad d_{36}=0$ & $E_{p l}=2 \mathrm{GPa}$ \\
$\beta_{31}=8 \times 10^{-4} \mathrm{pm}^{2} / \mathrm{V}^{2}$ & $v_{p l}=0.1$ \\
$E_{p}=63 \mathrm{GPa}$ & $t_{p l}=5 \mathrm{~mm}$ \\
$v_{p}=0.28$ & \\
$t_{p}=0.5 \mathrm{~mm}$ & \\
\hline
\end{tabular}




\section{Figures}

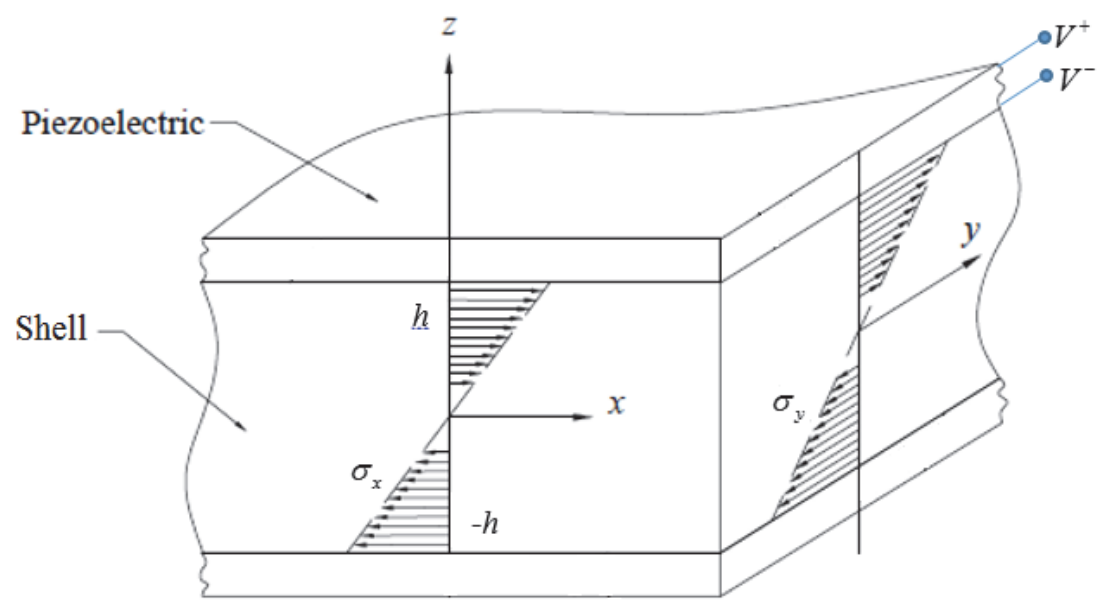

Figure 1. Stress distribution in the differential plate element due to active piezoelectric

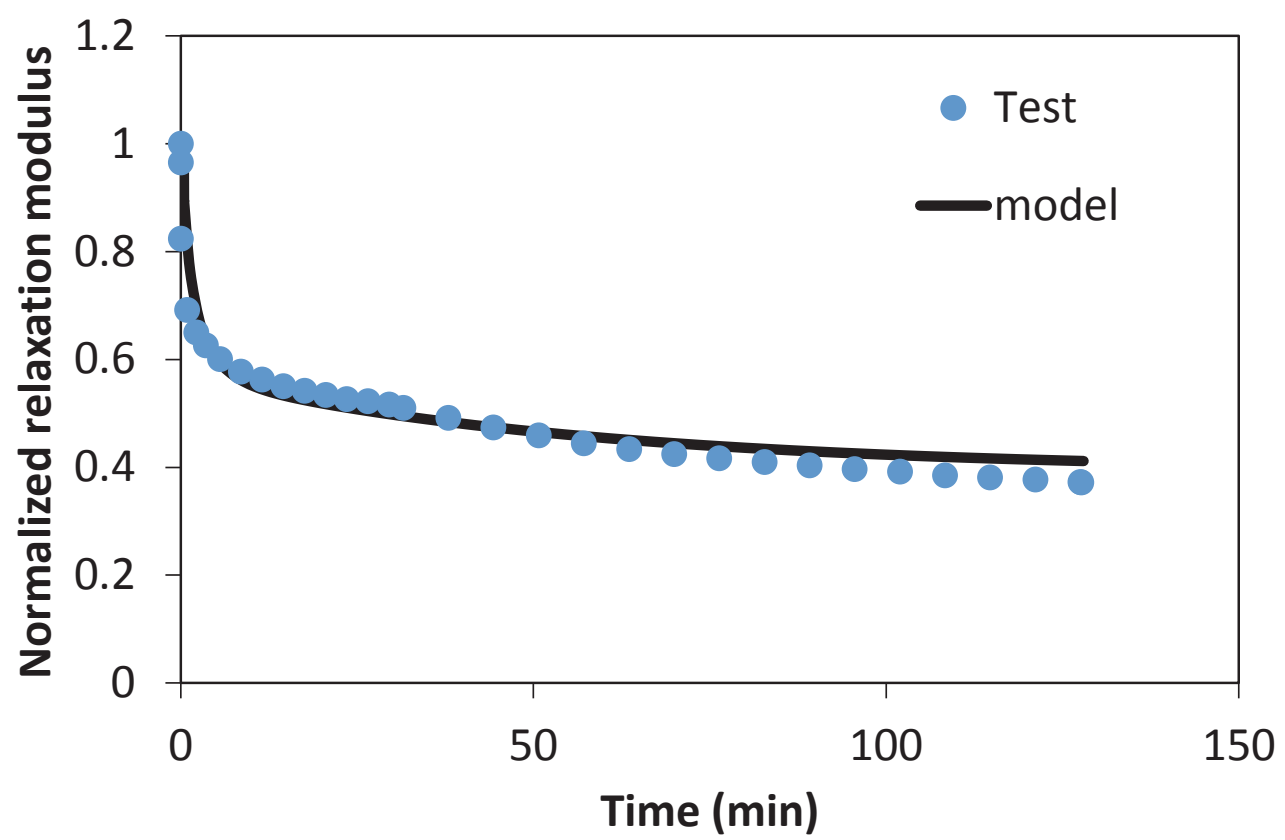

Figure 2. Time dependent relaxation function at room temperature 


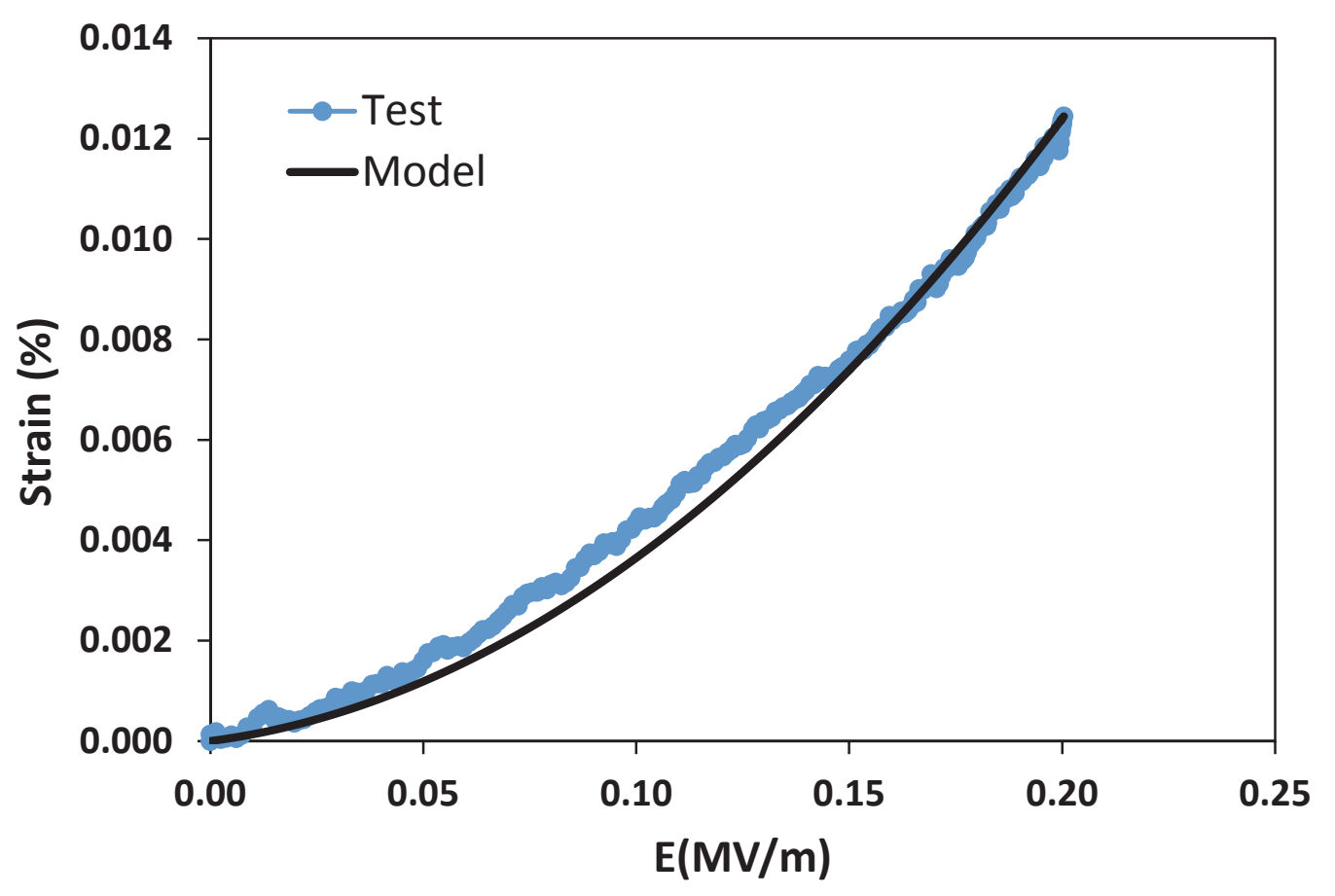

Figure 3. Strain response of AFC in fiber direction

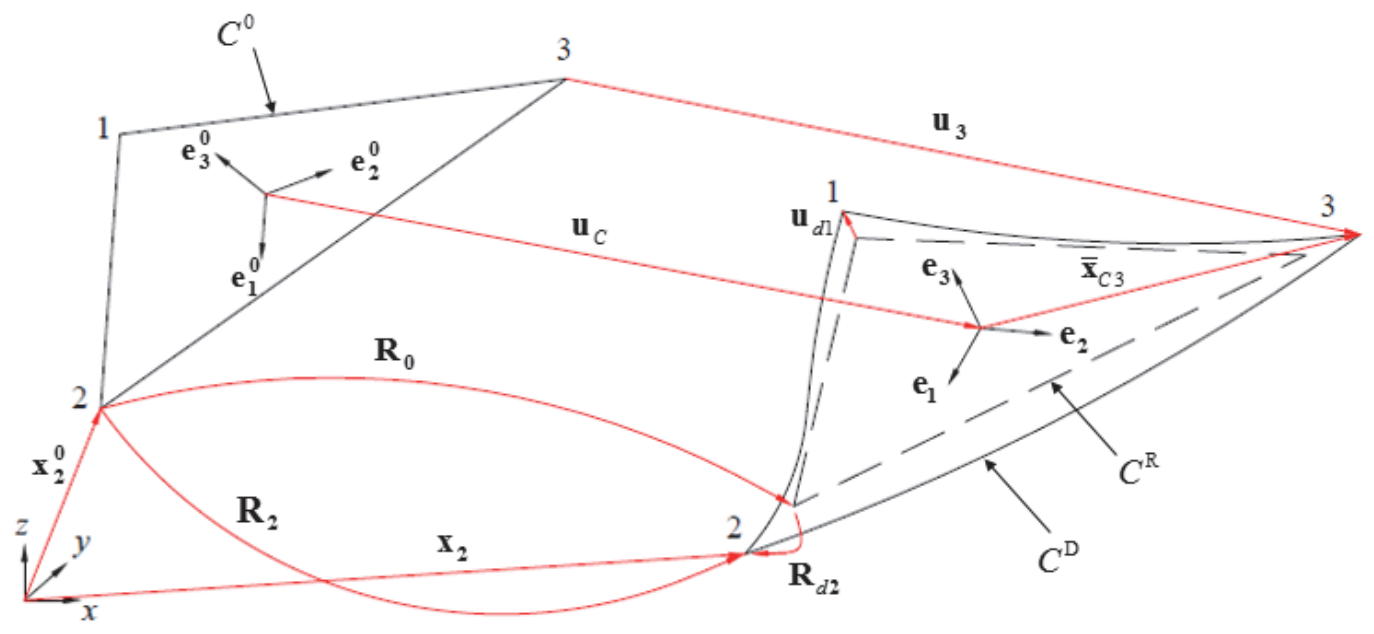

Figure 4. Co-rotational element kinematics 


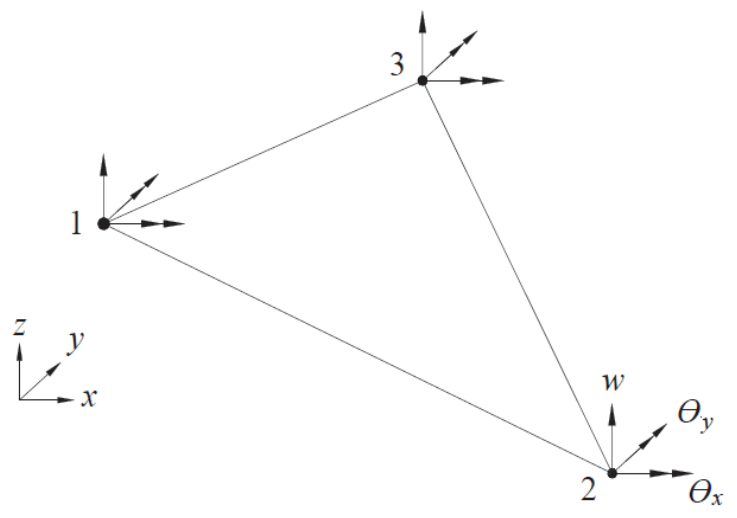

(a)

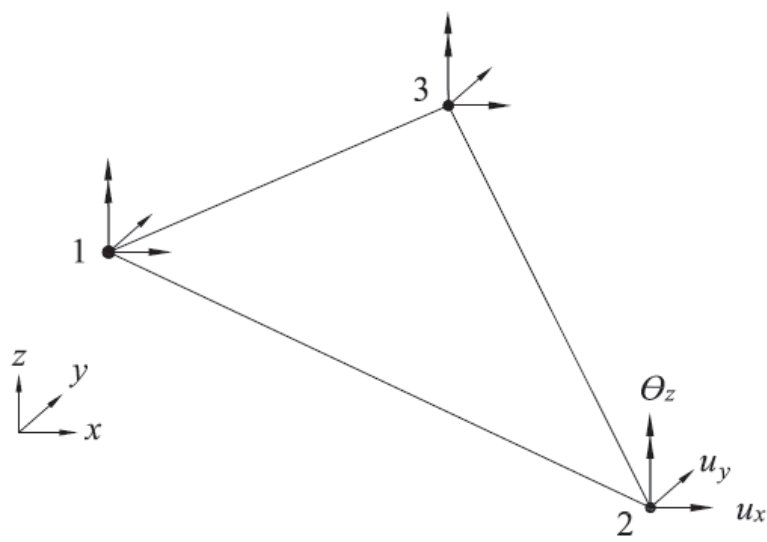

(b)

Figure 5. (a) Plate bending element and (b) Membrane element

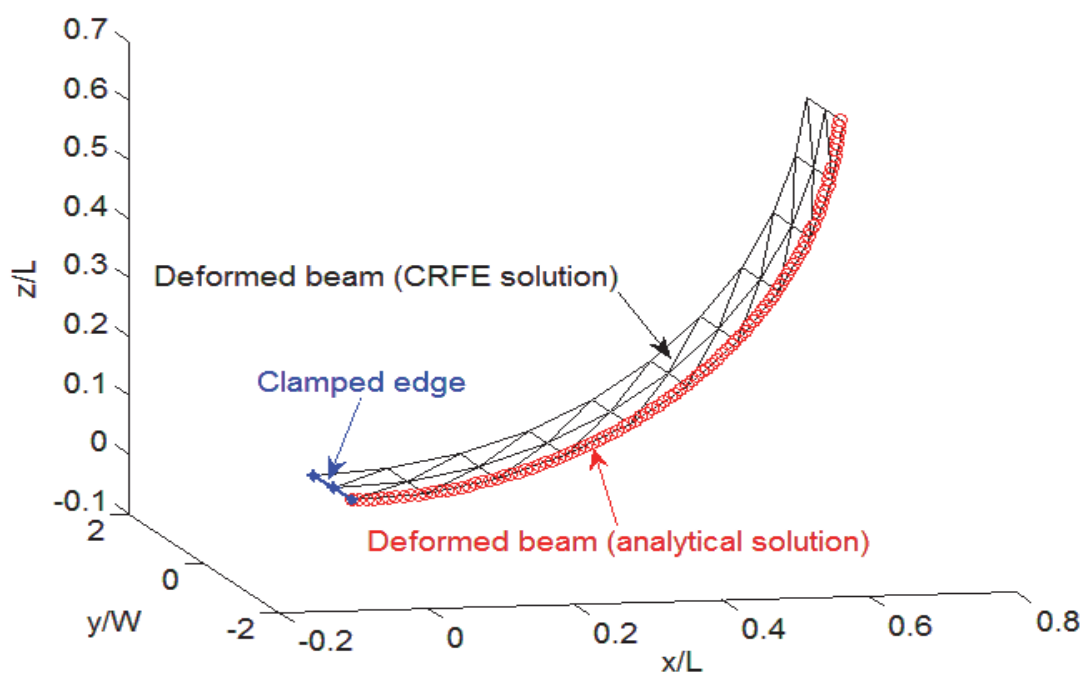

Figure 6. Comparison of predicted deformed configuration of the beam by CRFE method with Reissner's theory approach 


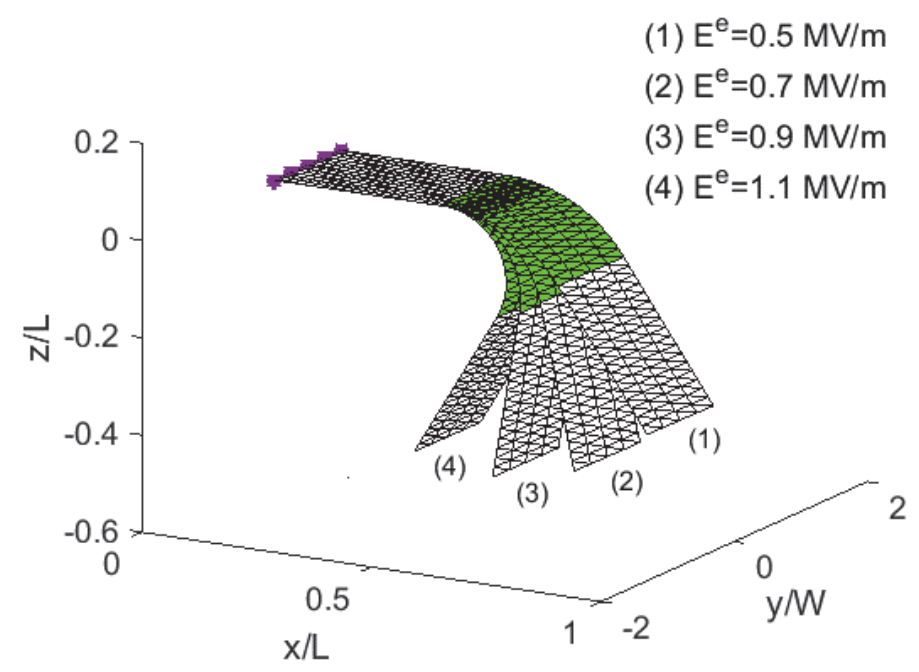

Figure 7. Configuration of the cantilever plate under various magnitude of electric stimulus

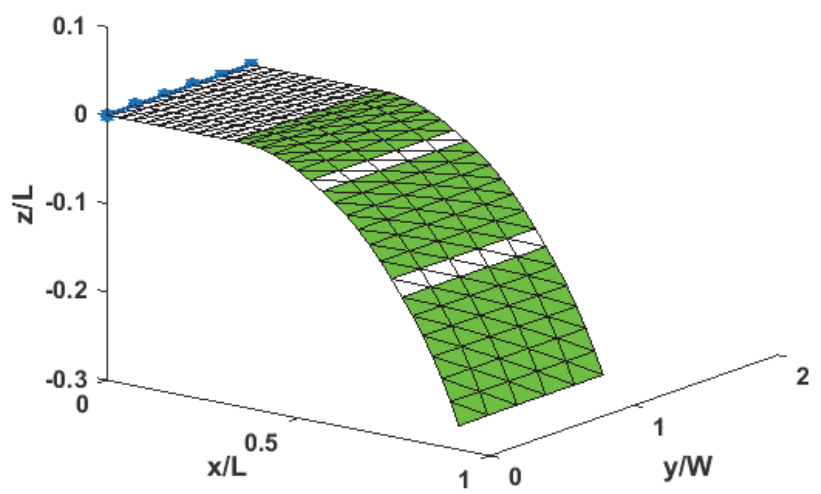

(a)

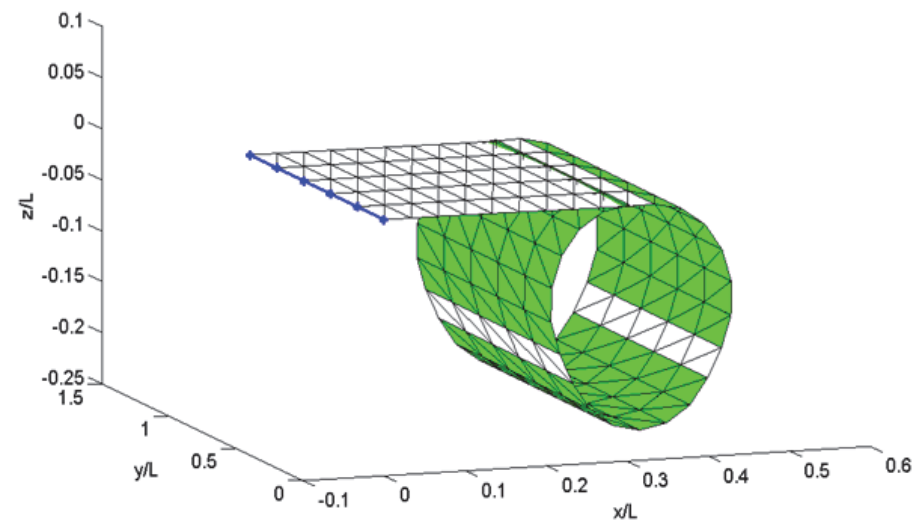

(b)

Figure 8. Configuration of a cantilever shell under actuation of three pairs of piezoelectric patches with (a) linear behavior (b) nonlinear behavior in terms of electric field 


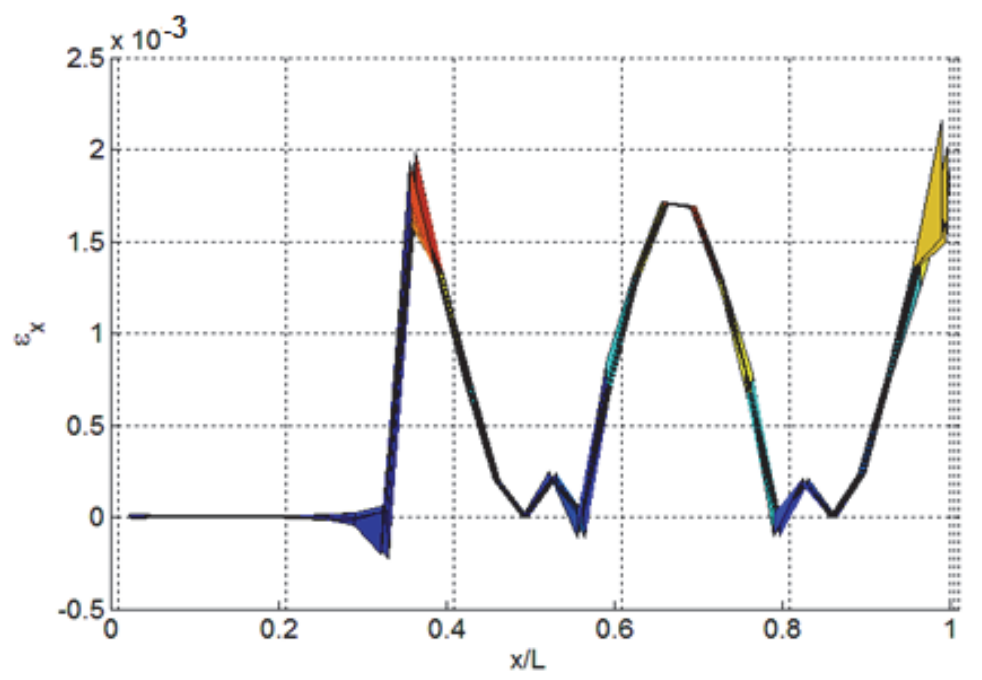

Figure 9. Axial strain distribution $\left(\varepsilon_{x}\right)$ of the cantilever shell under actuation of three pairs of piezoelectric patches nonlinear behavior in terms of electric field

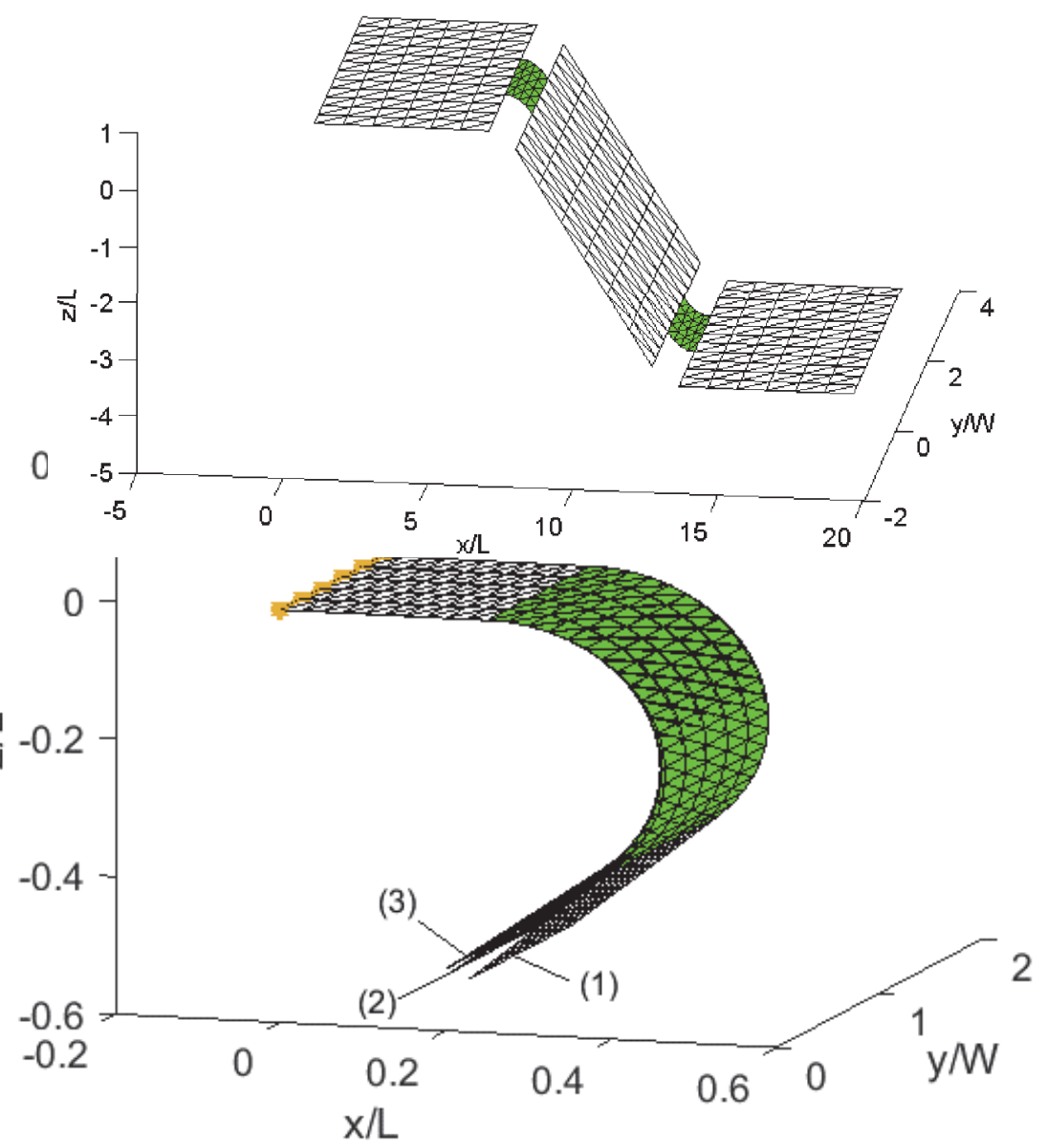

Figure 10. Configuration of the smart viscoelastic shell under actuation of one pairs of piezoelectric patches at different actuation time, $T$. 


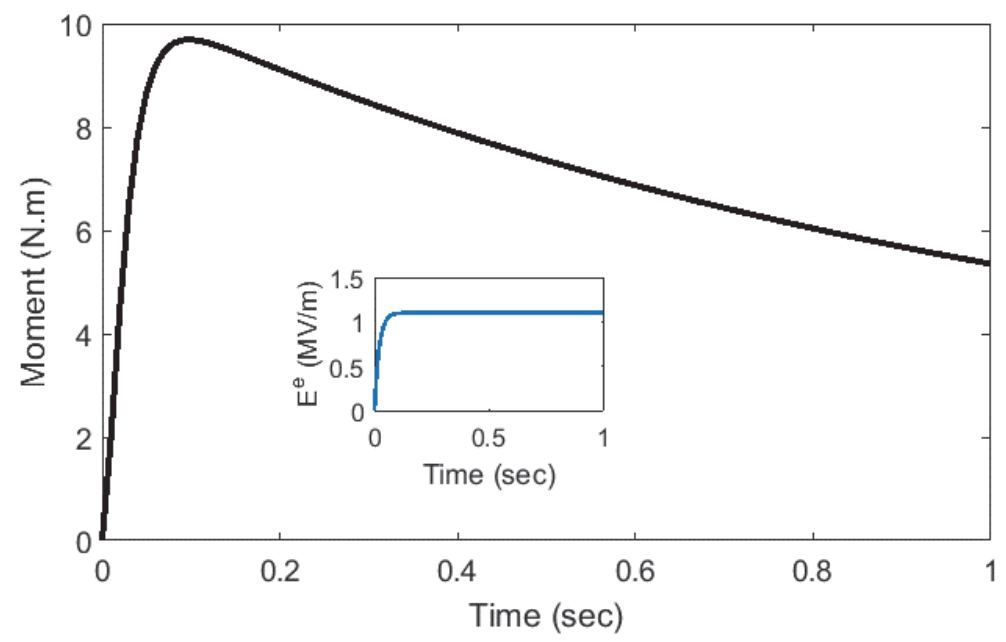

Figure 11. Induced moment to the substrate under the electric stimulus $E^{e}(t)$

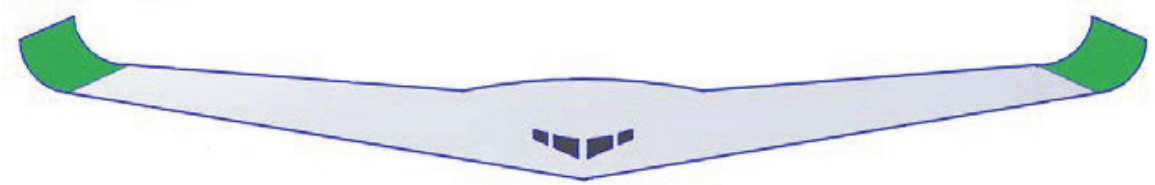

(a)

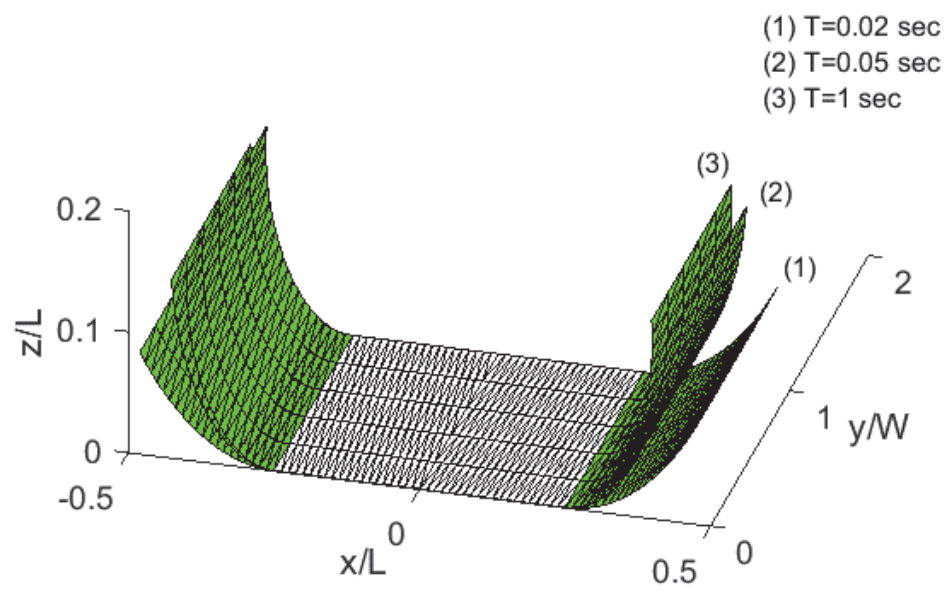

(b)

Figure 12. (a) A UAV concept and (b) configuration of the smart viscoelastic shell under actuation of two pairs of elastic piezoelectric patches at different actuation time, $\mathrm{T}$. 


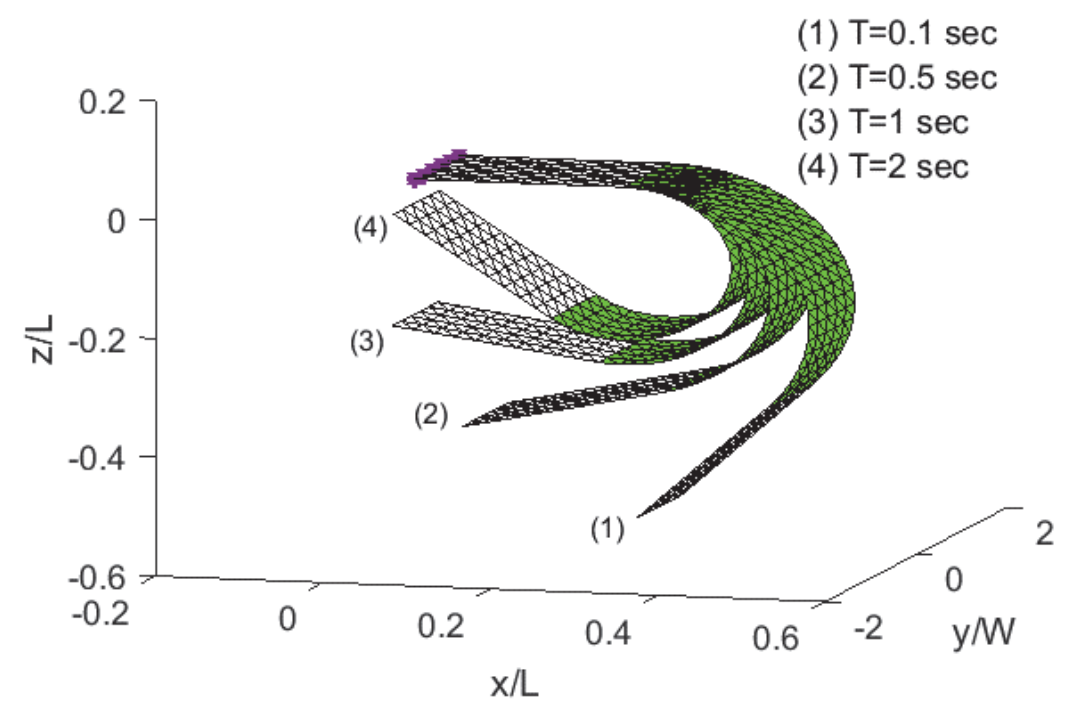

Figure 13. Configuration of the smart viscoelastic plate under actuation of one pair of AFC patches at different actuation time, $T$ 


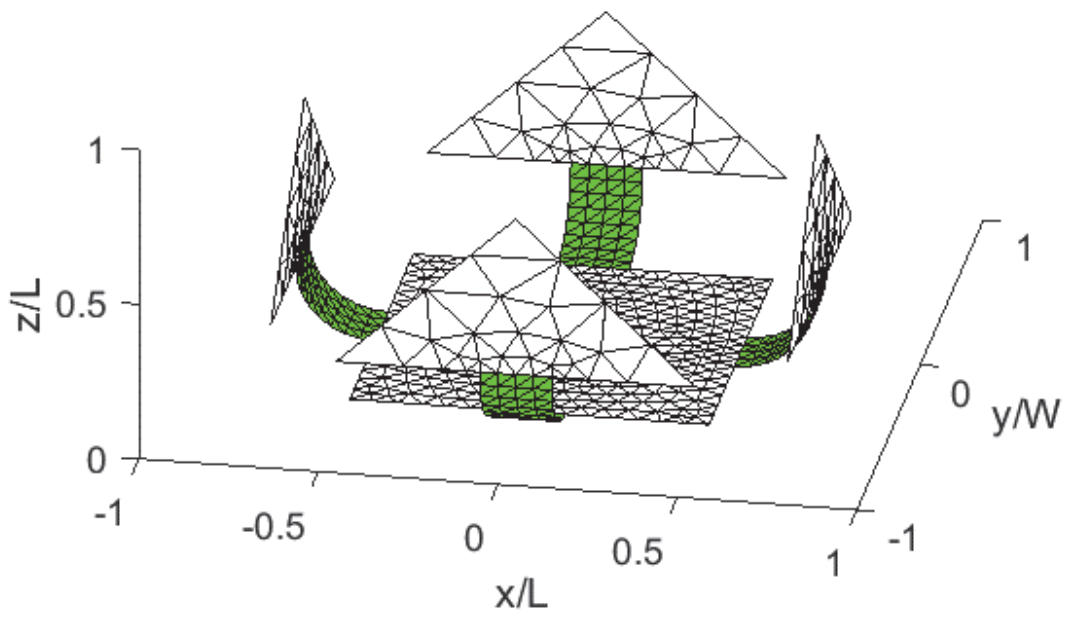

(a)

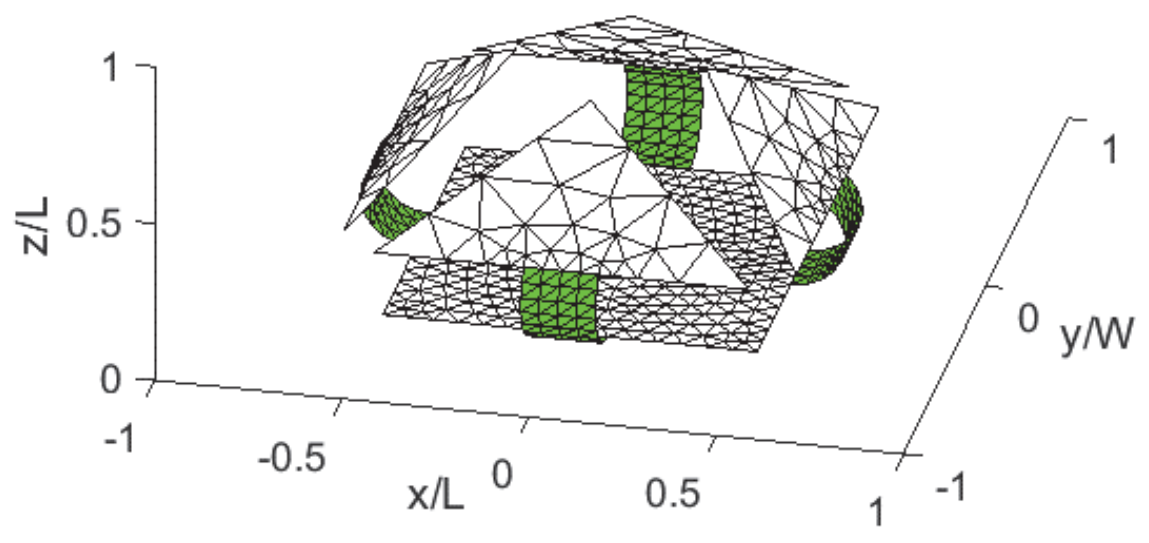

(b)

Figure 14. Configuration of the smart viscoelastic shell under actuation of four pairs of AFC patches at (a) $\mathrm{T}=0.1 \mathrm{sec}$ and (b) $\mathrm{T}=1 \mathrm{sec}$ 


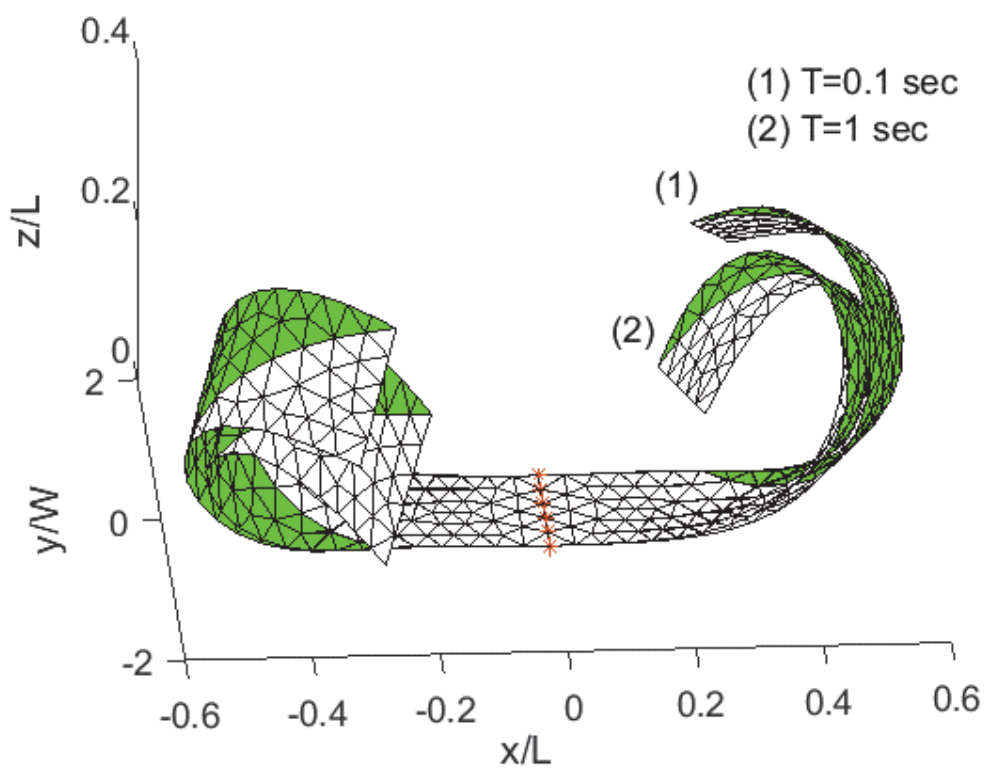

(a)

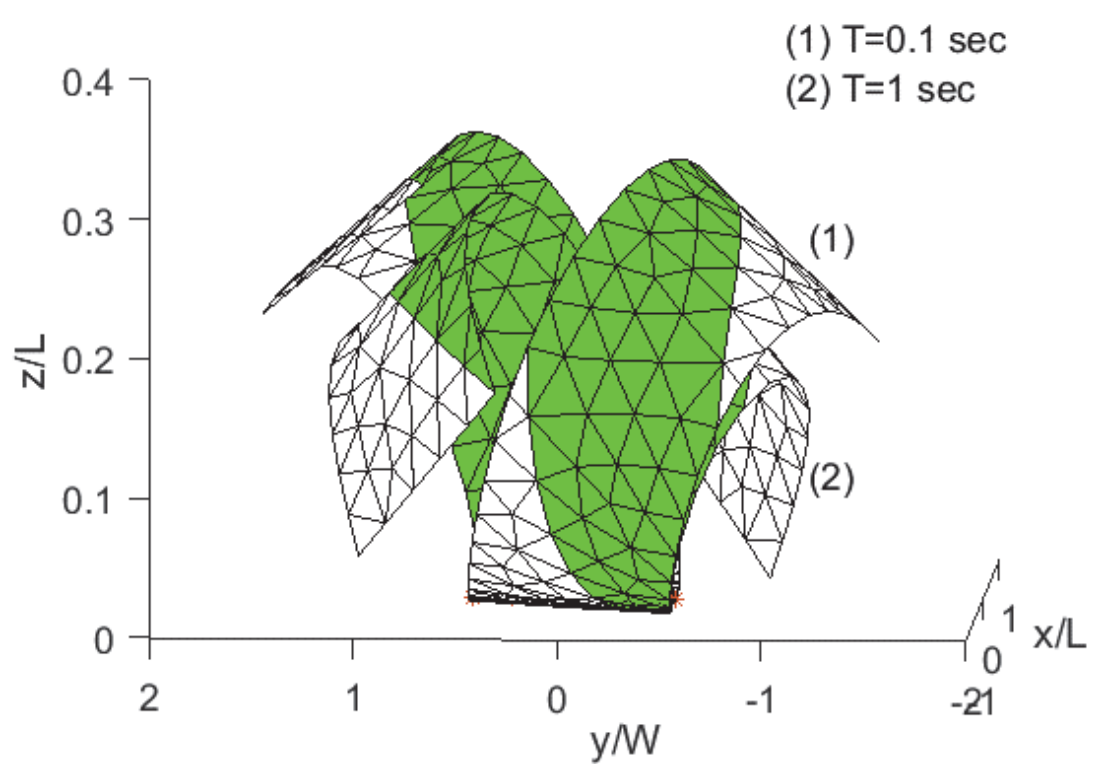

(b)

Figure 15. Configuration of the smart viscoelastic shell under actuation of two pairs of AFC patches at $\mathrm{T}=0.1 \mathrm{sec}$ and $1 \mathrm{sec}$ form two view angles $(\mathrm{a}, \mathrm{b})$ 


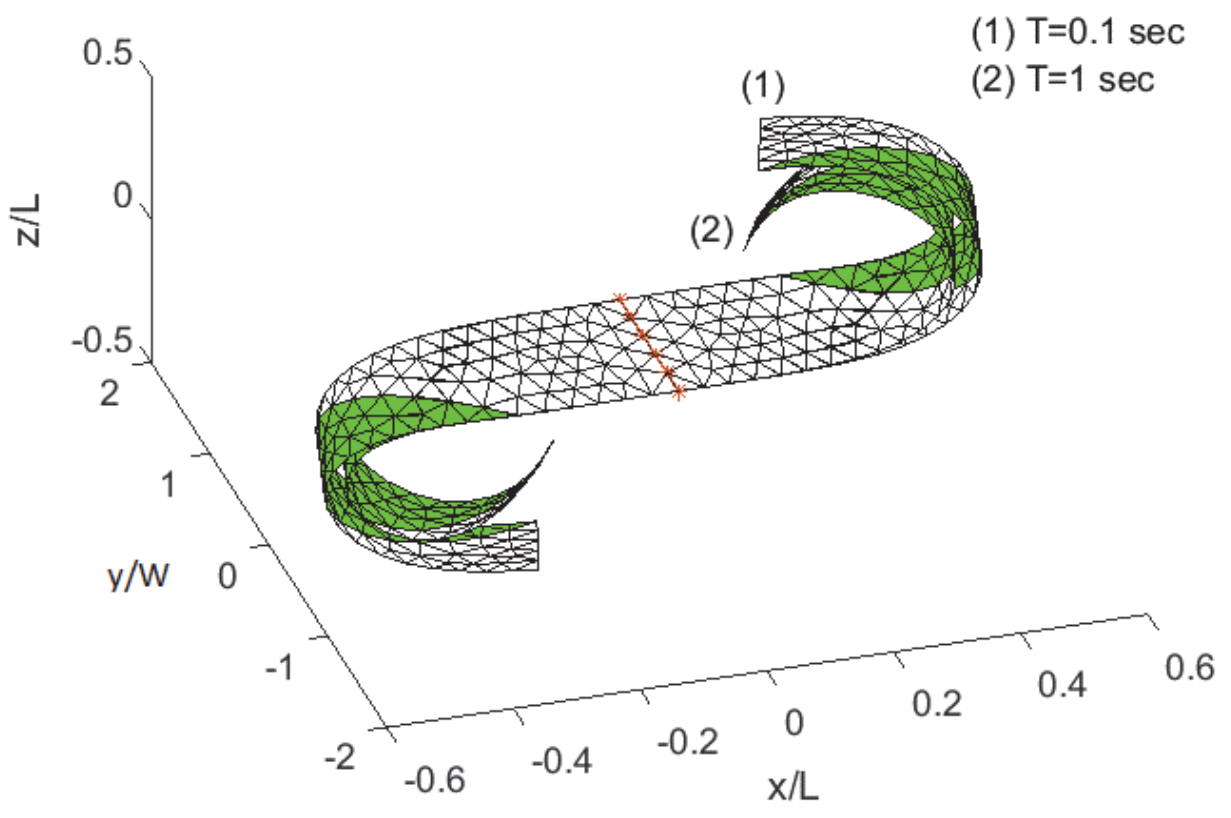

(a)

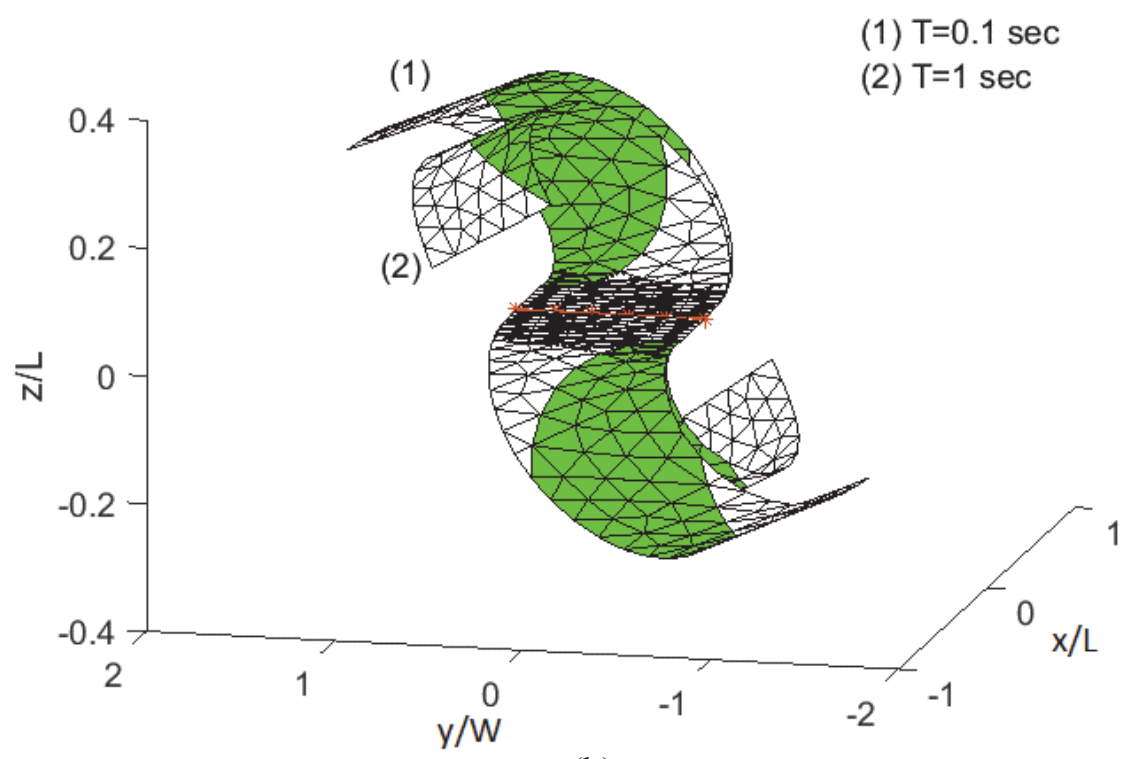

(b)

Figure 16. Configuration of the smart viscoelastic shell under actuation of two pairs of AFC patches in opposite directions at $\mathrm{T}=0.1 \mathrm{sec}$ and $1 \mathrm{sec}$ form two view angles $(\mathrm{a}, \mathrm{b})$ 